\title{
Cellulose Solutions in Water Containing Metal Complexes ${ }^{\dagger}$
}

\author{
Kay Saalwächter and Walther Burchard* \\ Institute of Macromolecular Chemistry, University of Freiburg, \\ Stefan Meier-Str. 31, 79104 Freiburg, Germany
}

\section{Peter KIufers, G. Kettenbach, and Peter Mayer}

Institute of Inorganic Chemistry, University of Munich, Butenandtstr. 5-13 81377 Munich, Germany

\section{Dieter Klemm and Saran Dugarmaa}

Institute of Organic Chemistry and Macromol ecular Chemistry, University of J ena, Humboldtstr. 10, 07743 J ena, Germany

Received November 8, 1999; Revised Manuscript Received March 23, 2000

\begin{abstract}
Aqueous solutions of a number of metal complexes have been found to dissolve cellulose. Recently, a number of new metal complexes have been developed that completely dissolve cellulose by deprotonating and coordinative binding the hydroxyl groups in the C2 and C3 position of the anhydro glucose. A detailed comparative light scattering study is given for cellulose in Schweizer's reagent (cuoxam), $\mathrm{Ni}$-tren, and Cd-tren. Cuoxam is the well-known solution of cupric hydroxide in aqueous ammonia, and the abbreviation tren stands for tris(2-aminoethyl)amine. Cuoxam and Ni-tren are deep blue solvents. The light scattering measurements were carried out with the blue line of an argon ion laser at wavelength $\lambda_{0}=457.9 \mathrm{~nm}$, and the data from these solvents required an absorption correction according to the Lambert-Beer law. Cd-tren is almost colorless, and the data could be used without correction. Because of traces of colloid particles, possibly originating from the metal hydroxides, a special treatment for optical clarification became necessary. A large number of samples, cotton linters, various pulp celluloses, and bacterial celluloses, were studied. All three solvents exhibited good solution properties, but only Cd-tren was capable of dissolving also the highest degrees of polymerization of cotton linters and bacterial cellulose $\left(D_{\mathrm{w}}=9700\right)$. The limits for the two other solvents were $\mathrm{DP}_{\mathrm{w}}<6300$ for Ni-tren and $\mathrm{DP}_{\mathrm{w}}<5300$ for cuoxam. A fairly high chain stiffness was found with Kuhn segment lengths of $I_{K}=15.8 \pm 1.4 \mathrm{~nm}$ for Cd-tren, $I_{K}=10.2 \pm 0.8 \mathrm{~nm}$ for $\mathrm{Ni}$-tren, and $\mathrm{I}_{\mathrm{K}}=13.1 \pm 1.2 \mathrm{~nm}$ for cuoxam, corresponding to characteristic ratios of $\mathrm{C}_{\alpha}=24.6,15.4$, and 19.4, respectively. The problem of preferential adsorption is discussed.
\end{abstract}

\section{Introduction}

Cellulose bel ongs to the ol dest macromolecules known to us. Staudinger's ${ }^{1}$ pi oneering work in the 20th century on macromol ecules started with this biopolymer. Still, after more than 70 years the structures of individual cellulose molecules remain not fully elucidated. This lack of knowledge is an effect of poor solubility even in uncommon solvents. To obtain information at least on the molar mass, Staudinger ${ }^{2}$ introduced the polymer analogous substitution of the $\mathrm{OH}$ groups, which were considered as the origin for strong aggregation, and applied it to cellulose. F or two fully substituted derivatives complete dissolution down to the molecular level was indeed obtained. These are cellul ose trinitrate and cellulose tricarbanilate which both are soluble in a large number of common organic solvents.

Besides appreciable efforts invested in the chemistry of derivatization, serious problems remained. Preparation of fully substituted derivatives turned out to be difficult, because the cellulose chains are embedded in a partially crystalline structure ${ }^{3}$ that cannot easily be broken up. Not fully substituted derivatives, on the other hand, were found to be strongly aggregated. ${ }^{4}$ The samples appear to possess a special supramolecular structure in solution that is reminiscent of the semicrystalline structure of the parental cellul ose. Because of these facts, scientists at no time ceased searching for

\footnotetext{
† Dedicated to Professor Pavel Kratochvil, Prague, on the occasion of his 70th birthday and in admiration of his skill in guiding a large Institute through a difficult period.
}

new solvents that are capable of dissolving cellulose directly and molecularly dispersed.

A first success was reported by Schweizer, ${ }^{5}$ who discovered that a solution of cupric hydroxide in aqueous ammonia has the power to dissolve cellulose. This solvent is often called cuoxam. We will use this abbreviation despite some criticisms that has been raised against this nomenclature. Suggestions for the mechanism of dissolution were made later. Accordingly, cuoxam coordinatively binds the deprotonated hydroxyl groups in the $\mathrm{C} 2$ and $\mathrm{C} 3$ positions of the anhydro glucose units (AGU). The validity of this suggestion was recently proven by systematic studies of crystal structures with oligosaccharides, combined with computer simulations and calculations of complex stability constants. ${ }^{6,7}$ Further confirmation was obtained from light scattering measurements. ${ }^{7,8}$ Figure 1 shows the structure of the complex bound to the AGU. Cuoxam has some disadvantages which consist of easy degradation of the cellul ose chain, a deep blue col or that inhibits exact light scattering studies, and a restricted dissolution power that is limited to degrees of polymerization of $\mathrm{DP}_{\mathrm{w}}<$ 5000. Furthermore, strong association occurs al ready in moderately concentrated solutions. ${ }^{9}$ The reason for the restricted solubility was recently disclosed ${ }^{6,7}$ but was not known when J ayme started a systematic search for other metal-based cellul ose solvents. In the following a special nomenclature is used, a summary of which is given in the Appendix.

$\mathrm{J} \mathrm{ayme}^{10}$ used ethylenediamine (en) and other polydentate ligands to form compl exes with metal ions such as $\mathrm{Cu}^{2+}, \mathrm{Ni}^{2+}, \mathrm{Cd}^{2+}, \mathrm{Fe}^{2+}$, and $\mathrm{Co}^{2+}$. His idea was that 
<smiles>COC1O[C@@H](CO)C(OC2OC(CO)C(OC3O[C@@H](CO)C(C)C(O)C3O)C(O)C2O)C(O)C1O</smiles>

$m \mathrm{Cu}\left(\mathrm{NH}_{3}\right)_{n}(\mathrm{OH})_{2}$ $-2 m \mathrm{H}_{2} \mathrm{O},-m(n-2) \mathrm{NH}_{3}$

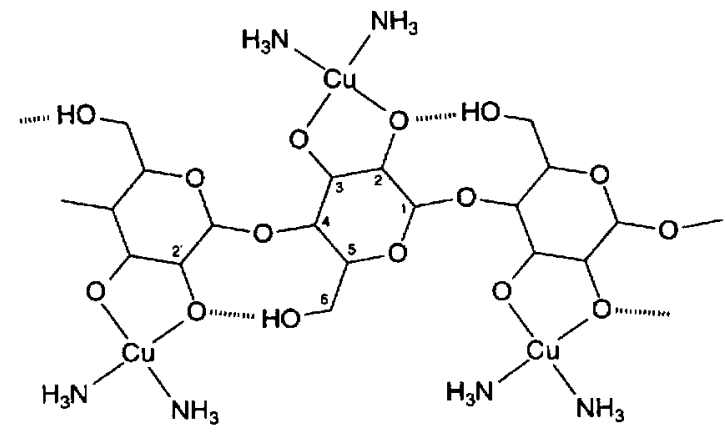

Figure 1. Part of the hydrogen-bonding system in native cellulose and its change after coordinative binding of copper in cuoxam at the deprotonated olate groups in the $\mathrm{O}_{2}^{-}$and $\mathrm{O}^{-}$position of the anhydro glucose unit (AGU). Note: the hydrogen bond between the primary $\mathrm{OH}$ group in the $\mathrm{C} 6$ position and the ring oxygen of the next AGU is interrupted, but the other hydrogen bond is enhanced. These bonds occur on both sides of the chain and cause an enhanced chain stiffness. ${ }^{7}$

these complexes should have a similar potential of dissolving cellulose as observed with cuoxam. In fact, all these reagents gave clear solutions indicating full solubility of the cellulose. The mechanism of dissolution, however, remained unclear. This question is presently the subject of research in inorganic chemistry. ${ }^{11}$ The results of the present investigation are based on exceptional dissolution mechanisms which are rare with synthetic polymers in common organic solvents. The mechanism appears applicable to various polysaccharides but also to other polar polymers. Therefore, the dissolution mechanism may be displayed here at some length before turning to the report of the solution properties of cellulose in three metal-based solvents.

\section{Dissolution Mechanism of Metal-Based Solvents}

Dissolution of cellulose by nonderivatizing solvents was recognized as being possible by two different mechanisms. In both cases the highly organized hydrogen-bonding system, which surrounds the cellulose chain in the fibrils, has to be broken up. The following consideration may for simplicity be confined to the intrachain hydrogen bonds which are effective between the primary $\mathrm{OH}$ group in the $\mathrm{C} 6$ position of an $\mathrm{AGU}$ and the $\mathrm{OH}$ group in the $\mathrm{C} 2$ position of the neighbored AGU. A second bond is formed on the other side of the stretched chain between the $\mathrm{OH}$ group in the $\mathrm{C} 3$ position of an AGU and the oxygen of the ring from the neighbored AGU. ${ }^{12}$ The hydrogen-bonding system is partially broken when deprotonating both $\mathrm{OH}$ groups in the $\mathrm{C} 2$ and $\mathrm{C} 3$ position.

However, the mere removal of protons by strong alkoxy acceptors is not sufficient to produce solubility because the cations, associated with anionic alkoxide, undergo Coulomb interactions with the deprotonated anion groups of different chains. Strongly associated ion pairs cause cross-linking and insolubility. ${ }^{11 a}$ Undesired cross-linking can be prevented by creating solvation separated ion pairs, and this can be achieved sterically by bulky cations, as for instance benzyltrimethylammonium hydroxide (Triton B) or tetraal kylammonia hydroxides. In these cases the cations are not fixed to a special position of the chain, and solubility is induced by common solvation procedures.

The other possibility consists of a strong coordinative binding of the cations where the two deprotonated $\mathrm{OH}$ groups in the C 2 and $\mathrm{C} 3$ position may act as a chelating ligand. This second mechanism is realized with cuoxam, as demonstrated by Figure $1 .{ }^{7}$ The two $\mathrm{NH}_{3}$ groups in this complex can be further replaced by two other deprotonated $\mathrm{OH}$ groups from an AGU of another chain which results in cross-linking. This Norman compound ${ }^{13}$ is a highly swollen gel and is induced by adding $\mathrm{NaOH}$ to the cuoxam solution of cellulose. Which reaction actually is observed depends on the stability constants of the corresponding chelates at different $\mathrm{pH}$. Careful spectroscopic measurements and derivation of stability constants for competing complexes with cuoxam ${ }^{7,14}$ showed that the equilibrium is shifted toward the Norman compound when the $\mathrm{pH}$ is increased beyond a critical value. It also is formed when at constant $\mathrm{pH}$ the concentration of cellulose is increased. This competition between two complexes explains the limited concentration range of solubility. It also explains the limiting degree of polymerization that can be dissolved, because Iong chains can fold back and intramolecular crosslinking can take place. ${ }^{8}$

In Cu-en [ $\left.\left(\mathrm{NH}_{2}-\mathrm{CH}_{2}-\mathrm{CH}_{2}-\mathrm{NH}_{2}\right) \mathrm{Cu}(\mathrm{OH})_{2}\right]$ the ammonia groups in cuoxam are replaced by ethylenediamine (en $)^{15}$ which causes deprotonation, but coordinative binding to the anionic oxygens in the C2 and C3 position of the glucose ring does not take place. The solvation power is probably solely based on Coulombic interactions of the charged glucose ring combined with a chain separating power of the cations.

This is not true for all ethylenediamine forming complex cations. An exceptional case is observed with the yellow palladium-based solvent $\mathrm{Pd}$-en, an aqueous solution of [(en) $\left.\mathrm{Pd}(\mathrm{OH})_{2}\right]$ that undergoes stable planar coordinative binding with the deprotonated diolate groups of the AGU in cellulose, in a manner that may justify denoting it as a derivatizing solvent. This fact was proven by complexes with oligosaccharides which formed well-defined crystal structures.7,16 Moreover, there is ${ }^{13} \mathrm{C}$ NMR evidence of virtually complete coordination to cellulose. Good solution behavior of this complex with cellulose was recently demonstrated by Ballauff ${ }^{17}$ with light scattering measurements from three samples.

In the search for other metal-based solvents for cellulose that are less expensive than $\mathrm{Pd}$-en, the $\mathrm{Ni}^{2+}$ cation appears as a suitable alternative to $\mathrm{Cu}^{2+}$. However, there are some problems regarding coordination equilibria in the case of nickel. Nill central atoms are coordinated strictly octahedrally to aminine and alkoxo/ hydroxo-type ligands, whereas square-planar configurations are favored with copper. Cellulose or low molecular weight diols may thus form coordination compounds of the general formula $\left[(e n)_{2} \mathrm{Ni}\left(\mathrm{LH}_{-2}\right)\right](\mathrm{L}=$ diol $)$ when ethylenedimamine is used to provide the proper number 

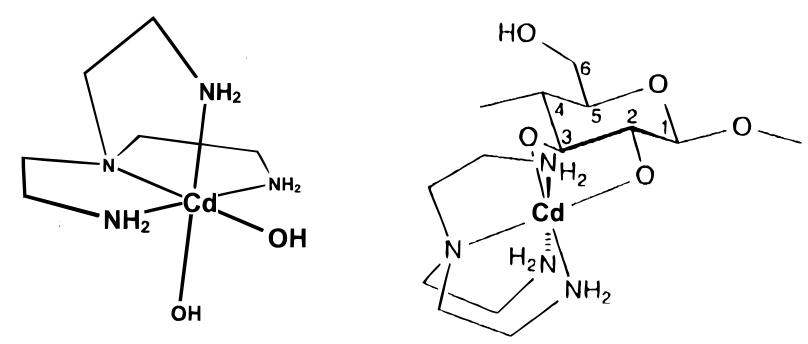

Figure 2. Chemical structure of the $\mathrm{Cd}$-tren $(\mathrm{OH})_{2}$ complex and its coordinative binding to the AGU in cellulose. ${ }^{11}$ [tren $\left.=\mathrm{N}\left(-\mathrm{CH}_{2}-\mathrm{CH}_{2}-\mathrm{NH}_{2}\right)_{3}\right]$.

of ligands to achieve the octahedral coordination of $\mathrm{Ni}^{\prime \prime}$. However, despite the particularly high stability of analogous [(en) $\left(\mathrm{Cu}\left(\mathrm{LH}_{-2}\right]\right.$ species, ${ }^{7}$ coordination equilibria in en/Ni/diol systems do not favor a species of this kind. Instead, the hypothetical coordination equilibrium

$$
\begin{aligned}
& \left.3 \text { “" }(\text { en })_{2} \mathrm{Ni}\left(\mathrm{LH}_{-2}\right)\right]^{\prime \prime} \leftrightarrow \\
& 2\left[\mathrm{Ni}(\mathrm{en})_{3}\right]^{2+}+\mathrm{Ni}(\mathrm{OH})_{2}+3 \mathrm{~L}+4 \mathrm{OH}^{-}
\end{aligned}
$$

is shifted to the right in the case of ethylenediamine as nitrogen ligand. The formation of a $\mathrm{NiN}_{6}$-type complex and precipitation of $\mathrm{Ni}(\mathrm{OH})_{2}$ can be prevented by using a tetradentate chelate ligand as for instance tris(2aminoethyl )amine (tren). ${ }^{11 a}$ The corresponding complex leaves two cis-configurated positions at the $\mathrm{Ni}$ octahedron free for coordinative binding of the two diolate moieties of the AGU in cellulose. The Ni-tren complex has another advantage of possessing a bulky, rather inert back, thus supporting chain separation by steric interaction. A very similar complex structure is obtained with $\mathrm{Cd}^{2+}$ (Cd-tren), which has only a weak yellow color in contrast to $\mathrm{Ni}$-tren which is deep blue. The two metal complexes of Ni-tren and $\mathrm{Cd}$-tren, $\left[\mathrm{Ni}(\right.$ tren $\left.)(\mathrm{OH})_{2}\right]$ and $\left[\mathrm{Cd}(\operatorname{tren})(\mathrm{OH})_{2}\right]$, turned out to be efficient in dissolving cellulose down to the molecular level. Figure 2 shows the structure of the free complex and in its coordinatively bound state.

In the following we examine the solution properties of cellulose in these two new metal-based solvents and compare these with the corresponding properties in cuoxam. As al ready mentioned, the study was based on a rigorous investigation of the coordinatively bound structures which were first obtained from crystal structures with suitable oligosaccharides in these solvents. In a further step this knowledge was tentatively generalized by assuming that similar mechanisms may be effective also with the AGU in cellulose. ${ }^{11}$ The validity of such considerations was first shown with cellulose in cuoxam 7,8 and led to a sensible explanation of the observed chain stiffness in this solvent. ${ }^{7}$ We were interested in learning whether coordinative binding of a metal complex to chains would significantly change common solution properties of polymers. We report light scattering results on molar mass $\mathrm{M}_{\mathrm{w}}$, radius of gyration $R_{g}$, second virial coefficient $A_{2}$, and Kuhn segment length $I_{K}$ which describes chain stiffness. In addition, viscosity measurements were performed, and the molar mass dependencies of $R_{g}, R_{h}, A_{2}$, and the intrinsic viscosity $[\eta]$ were examined. The results from the three solvents $\mathrm{Ni}$-tren, Cd-tren, and cuoxam are compared.

\section{General Remarks on Problems with Metal Complexing Solvents}

Characterization of linear polymers in organic solvents has become routine work in polymer analysis.
Experimental techniques and methods of data interpretation are well developed. A very different situation is encountered, however, with biopolymers, notably with polysaccharides. The many hydroxyl groups on the chains are capable of uncontrolled hydrogen bonding which often leads to strong association and formation of complex structures. These facts make it necessary to use uncommon solvents. Application of the metal-based solvents impose unanticipated problems. The solvents are often deeply colored, and reliable molar mass determination by light scattering was for a long time considered as not feasible. Only recently reasonable results were obtained for cellulose in cuoxam after applying an absorption correction. ${ }^{8,9}$ Nonetheless, reproducible and correct results of the molar mass remain difficult to attain. This has the following reason.

As is well-known, the molar mass of a polymer can be obtained by extrapolating the static light scattering data to zero scattering angle (forward scattering) according to the equation

$$
\left[\frac{\mathrm{R}_{\theta=0}}{\mathrm{C}}\right]_{\mathrm{C} \rightarrow 0}=\mathrm{KM}_{\mathrm{w}}
$$

in which $\mathrm{K}$ is an optical constant that describes the contrast of the polymer to the solvent. It contains as essential parameter the refractive index increment $\partial \mathrm{n} /$ $\partial \mathrm{c}$ and is given by the equation

$$
K=\frac{16 \pi}{\lambda_{0}^{4} N_{a}} n_{0}^{2}\left(\frac{\partial n}{\partial c}\right)^{2}
$$

where $\lambda_{0}$ is the wavelength of the primary beam light, $\mathrm{N}_{\mathrm{a}}$ Avogadro's number, and $\mathrm{n}_{0}$ the refractive index of the solvent. The refractive index increment is commonly measured in special differential refractometers, in which the refractive index of the solution $\mathrm{n}$ is directly compared with that of the solvent. Mostly this type of measurement gives the correct result. However, with the metal complexing solvents a yet nonpredictable effect is obtained by this procedure. A nonnegligible amount of the metal complexing agent is found being bound to the cellulose chain and increases the refractive index of the chain. Simultaneously, the refractive index of the surrounding solvent is slightly decreased. Thus, the differential refractometer measures an refractive index increment that can be considerably smaller than is actual ly effective in light scattering. The problem can be circumvented when an equilibrium dialysis of the solution against the solvent is performed, and the difference in the refractive indices between these two components is measured. ${ }^{18}$ Unfortunately, this procedure is not applicable to cel lulose sol utions because the membranes in dialysis chambers are based on cellul ose or cellulose acetate and become destroyed by the solvent.

Another technique would be the laborious determination of preferential adsorption of the metal compound to the chain as described in detail, e.g., by Strazielle ${ }^{19}$ in the book of Hugglin ${ }^{18}$ on light scattering. ${ }^{20}$ This method is involved with large errors but would be still of interest, because it would give us some idea how many metal compounds are bound to the chain. We refrained from this technique mainly because of the sensitivity of the solvents against air. In addition, we wondered whether the coordinative binding can be described by simple adsorption. It appears conceivable that as a consequence of coordination the cellulose 


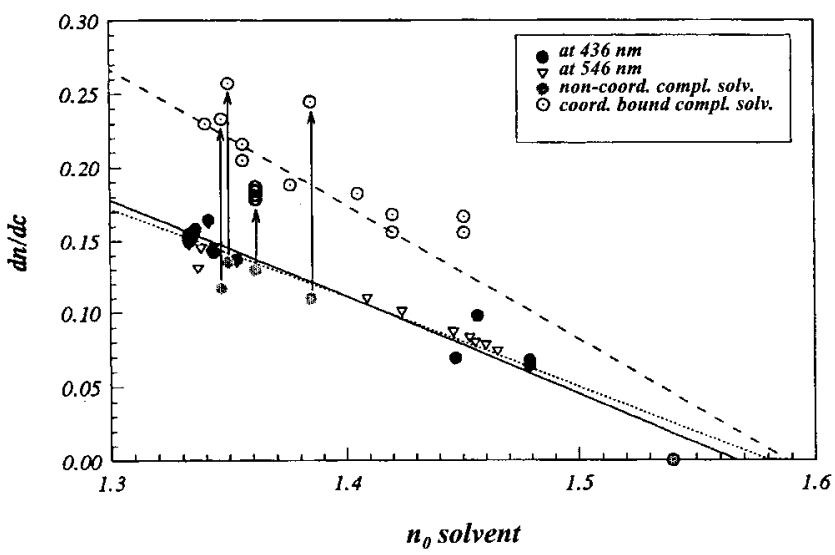

Figure 3. Refractive index increment dn/dc of glucans as a function of the solvent refractive index. Full line: noncoordinative binding at $\lambda_{0}=436 \mathrm{~nm}$, dotted line at $\lambda_{0}=546 \mathrm{~nm}$; dashed line coordinative binding, at $\lambda_{0}=436 \mathrm{~nm}$ in metalbased solvents. The arrows indicate the change due to cooperative binding. No difference between the values from different glucans was assumed (see text).

changes its character and thus the refractive index in a nonadditive manner.

Gralén ${ }^{21}$ solved the problem of the correct refractive index increment for cuoxam by forming a sharp boundary between solution and solvent in an ultracentrifuge. The difference in the refractive indices was then measured by Schlieren optics. This technique is not particularly accurate because the required sharp boundary is difficult to perform; it soon becomes diffuse due to commencing diffusion. Still our first results ${ }^{8,9}$ were obtained with Gralén's value.

Another estimate can be made from the dependence of $\mathrm{dn} / \mathrm{dc}$ on the solvent refractive index. Figure 3 shows the result for starch in several solvents. ${ }^{18}$ The same dependence may be assumed to hold also for cellulose because in both examples the anhydro glucose is a repeating unit. Slight differences are possible but are unlikely, because the same values for $\mathrm{dn} / \mathrm{dc}$ were found in previous studies for cellulose and amylose tricarbanilates. ${ }^{22}$ The refractive index increment for a special solvent now can be found by measuring the refractive index of the solvent and reading off from the graph the corresponding value for dn/dc. However, this value is somewhat too low if applied to the metal complexing solvents, because the refractive index of complexed cellulose is not that for cellulose but is enlarged by the bound metal complex. To find the factor, we prepared the tricarbanilate derivative for a selected number of celluloses and determined the degree of polymerization. The polymer analogous carbanilation is a long known technique, ${ }^{22}$ where all the measured chain properties gave no indication for degradation. This value for the degree of polymerization then was compared with that found for the cellulose in the metal complexing solvent using the intrapolated $\mathrm{dn} / \mathrm{dc}$. The factors thus evaluated for cuoxam, $\mathrm{Ni}$-tren, and $\mathrm{Cd}$-tren are given in Table 1. The standard deviation for theses data is fairly high and may be estimated to be about $\pm 15 \%$. Most reliable results were obtained with Cd-tren. No absorption correction had to be applied to the almost colorless solvent which improved the accuracy of light scattering data. The uncertainty is highest for $\mathrm{Ni}$-tren because of the deep blue color which required an absorption correction by a factor $3.25 \pm 0.25$.
Table 1. Refractive Index $n_{0}$, Viscosity $\eta_{0}$ (cPa s), and Density d $\left(\mathrm{g} / \mathrm{cm}^{3}\right)$ of Three Metal Complexing Solvents, Refractive Index Increment dn/dc $(\mathrm{mL} / \mathrm{g})$ for Cellulose in These Solvents, and Correction Factor $f$ a

\begin{tabular}{lccccc}
\hline solvent & $\mathrm{n}_{0}$ & $\mathrm{dn} / \mathrm{dc}$ & $\eta_{0}$ & $\mathrm{~d}$ & correction factor $\mathrm{f}$ \\
\hline Cd-tren & 1.347 & 0.233 & 1.384 & 0.9143 & $1.47 \pm 0.23$ \\
Ni-tren & 1.373 & 0.199 & 2.284 & 1.0891 & $1.35 \pm 0.21$ \\
cuoxam & 1.375 & 0.198 & 2.090 & 1.1172 & $1.62 \pm 0.16$
\end{tabular}

a The measured molar masses have to be multiplied with this correction factor to obtain the same degrees of polymerization as were found for the cellulose tricarbanilate of the same samples. The factor is a measure for the binding strength to the anhydro glucose.

\section{Experimental Section}

Solvents. Cuoxam (0.1 M). A $500 \mathrm{~mL}$ aliquot of ice-cooled $25 \%$ aqueous ammonia solution was added under argon atmosphere to $5 \mathrm{~g}(\sim 50 \mathrm{mmol})$ of $\mathrm{Cu}(\mathrm{OH})_{2}$ (Aldrich, technical grade). The solution was stirred for about $1 \mathrm{~h}$ and then stored at $6{ }^{\circ} \mathrm{C}$ in a refrigerator. The solution should be used for measurement within 1 week. Large colloids sensitively disturb light scattering measurements. Precipitated $\mathrm{Cu}_{2} \mathrm{O}$ caused no serious problems; these traces could be removed by ultracentrifugation of the solutions immediately before measurement.

Cd-tren $(0.8 \mathrm{M})$. A $102.3 \mathrm{~g}(0.8 \mathrm{~mol})$ sample of CdO (p.a. $>99.5 \%$, Fluka) was dispersed in $140.39 \mathrm{~g}$ of freshly distilled tris(2-aminoethyl)amine (tren, pract. $>95 \%$, Fluka). The boiling temperature at oil pump vacuum is about $80-100{ }^{\circ} \mathrm{C}$. Distillation did not alter the light yellow color. Mixing should be made gradually under stirring. After 10 min $\mathrm{H}_{2} \mathrm{O}$ was added and filled to about $0.9 \mathrm{~L}$. The solution was further stirred at room temperature until a clear solution was obtained. This usually occurred after a few hours, but depending on the quality of the used $\mathrm{CdO}$, sometimes up to 1 week was required. Storing the solutions at $4{ }^{\circ} \mathrm{C}$ in the refrigerator accelerated the dissolution process. Remaining traces of $\mathrm{CdO}$ were re moved by filtration through sintered glass filters. Finally, by adding water the solution was diluted to $1 \mathrm{~L}$. The sol ution still had a slight yellow color; this gradually disappeared when storing the solution in the refrigerator. After 3 weeks the col or was so weak that no absorption correction of the light scattering intensities was needed. Traces of precipitant were removed by filtration immediately before performing light scattering measurements. Cd-tren is highly alkaline, and access of $\mathrm{CO}_{2}$ should be avoided.

$\mathrm{Ni}$-tren $(0.9 \mathrm{M})$. A $116.3 \mathrm{~g}(0.4 \mathrm{~mol})$ sample of $\mathrm{Ni}\left(\mathrm{NO}_{3}\right)_{2} \cdot 6 \mathrm{H}_{2} \mathrm{O}$ was dissolved in $800 \mathrm{~mL}$ of $\mathrm{H}_{2} \mathrm{O}$. A $33.6 \mathrm{~g}(0.84 \mathrm{~mol})$ sample of $\mathrm{NaOH}$ dissolved in $400 \mathrm{~mL}$ of $\mathrm{H}_{2} \mathrm{O}$ was added under gentle stirring whereupon nickel oxide is formed as a voluminous green precipitant. The precipitant was centrifuged, washed with water, and centrifuged again. The procedure had to be repeated 5-6 times until no nitrate could be detected. The total amount of nickel oxide then was dispersed in a solution of 61.4 $\mathrm{g}(0.42 \mathrm{mmol})$ freshly distilled tris(2-aminoethyl)amine (tren, pract., Fluka). A $100 \mathrm{~mL}$ aliquot of $\mathrm{H}_{2} \mathrm{O}$ were added and stirred for $2 \mathrm{~h}$ at $50{ }^{\circ} \mathrm{C}$, avoiding access of oxygen. A deep blue color devel oped within a short time. The solution was stirred at room temperature for a further $24 \mathrm{~h}$. Excess nickel hydroxide was removed by centrifugation, and $\mathrm{H}_{2} \mathrm{O}$ was added to an amount of $500 \mathrm{~mL}$. The deep blue solution is strongly alkaline and absorbs $\mathrm{CO}_{2}$ but otherwise can be kept in a refrigerator for a long time without loss of quality.

Preparation of Solutions for Light Scattering. A different dissolution and darification procedure had to be developed for each solvent system.

Cuoxam. Cellulose in cuoxam is sensitive to light and air. To avoid chain degradation during dissolution, each concentration was dissolved separately in brown $25 \mathrm{~mL}$ flasks and kept under protecting argon gas atmosphere. All flasks were flushed with argon before filling. The polymer concentration ranged from 0.2 to $5 \mathrm{mg} / \mathrm{mL}$. The solvent was filtered through Anotop ceramic membrane filters of $0.02 \mu \mathrm{m}$ pore size (Merck) directly into the flasks using a special filter setup that was driven by 
a certain pressure of nitrogen gas. The dissolution time was kept as short as possible. In all cases the solutions were prepared in the morning and measured on the same day. Despite all protections, a noticeable degradation of the cellulose chain about $10 \%$ was observed by repeated light scattering measurement when keeping a solution overnight. The cotton linters samples became dissol ved al ready after half an hour; for the bacterial cellulose samples and some of the pulp celluloses a somewhat longer time was needed. In all cases a "fl oating check" was made to prove compl ete solubility (see section Optical Clarification of Solutions). The solutions then were pipetted into cylindrical light scattering cells, covered by argon gas, and tightly sealed with a stopper that was wrapped by Teflon ribbon and Parafilm.

$\mathrm{Ni}$-tren. For preparation of the solution in $\mathrm{Ni}$-tren no filtration of the solvent was required. Since no degradation of cellulose was found within 1 day, the solutions safely could be prepared the day before measurement. A dilution series of five concentrations in the same range as for the cuoxam solutions was made and transferred into the scattering cells. Further treatment before measurement is described in the next paragraph.

Cd-tren. The Cd-tren solvent contained a considerable amount of large, almost gellike colloidal particles that were visible by eye in the laser light. These particles could not be removed by centrifugation, because of the small difference in density between the highly swollen particles and the solution. Therefore, the solvent was filtered through Anotop ceramic membrane filters of $0.02 \mu \mathrm{m}$ pore size. The solutions were prepared as described for $\mathrm{Ni}$-tren. The dissolution process of cellulose was appreciably accelerated by cool ing the solutions to $-15^{\circ} \mathrm{C}$. A $10 \%$ degradation of the cellulose was observed when the solutions was stirred overnight. Therefore, the solutions were prepared in the morning and measured the same day.

Optical Clarification of Solutions. The final optical darification for the light scattering measurements was achieved by ultracentrifugation applying the floating technique of Dandliker. ${ }^{23}$ To this end the sealed LS cells were put in the $\mathrm{UZ}$ beakers of a swinging bucket rotor that were filled with a concentrated solution of $\mathrm{K}_{2} \mathrm{HPO}_{4}$. The concentration of the aqueous salt solution was increased until the LS cell started to float. The floated cells were centrifuged at $12000 \mathrm{rpm}$. A preparative ultracentrifuge L5-50B (Beckman, USA) was used. In all cases a floating check was made; i.e., the cell was centrifuged for different times, and the solutions were checked whether there was a change in the scattering intensity. If a considerable amount of material was removed by centrifugation, the curves in Zimm plots of the scattering intensities were shifted to larger values.

No change was observed with $\mathrm{Ni}$-tren and Cd-tren; only the colloidal hydroxides were removed. The colloids seriously disturb the angular dependence of the scattered light and cause a strong downturn in the Zimm plot at small angles. This effect disappeared after centrifugation. A different situation was observed with the cuoxam solutions. With freshly prepared solutions the effect of centrifugation was the same as for the two other solvents. No change was observed after centrifugation for 20 and $40 \mathrm{~min}$, respectively. However, when the solution after LS measurements was centrifuged again, the described shift of the curves in the Zimm plot occurred. Figure 4 shows an example. Apparently removed colloidal particles were formed again after a certain time. Possibly these particles adsorb a considerable amount of cellulose, and these aggregates are removed by centrifugation. Because of the low viscosity of cuoxam, solutions could be clarified already after 30-45 min of centrifugation. For Cd-tren and $\mathrm{Ni}$-tren solutions a much longer centrifugation time of about 100 min was required. Figure 5 demonstrates with two examples of Zimm plots the accuracy that was obtained.

Light Scattering Measurements. Solvent Parameters. Table 1 gives a list of solvent parameters. The refractive indices and refractive index increments are needed for the calculation of the contrast factors $\mathrm{K}$ in eq 2 . The refractive index is also required for calculating the light wavelength in

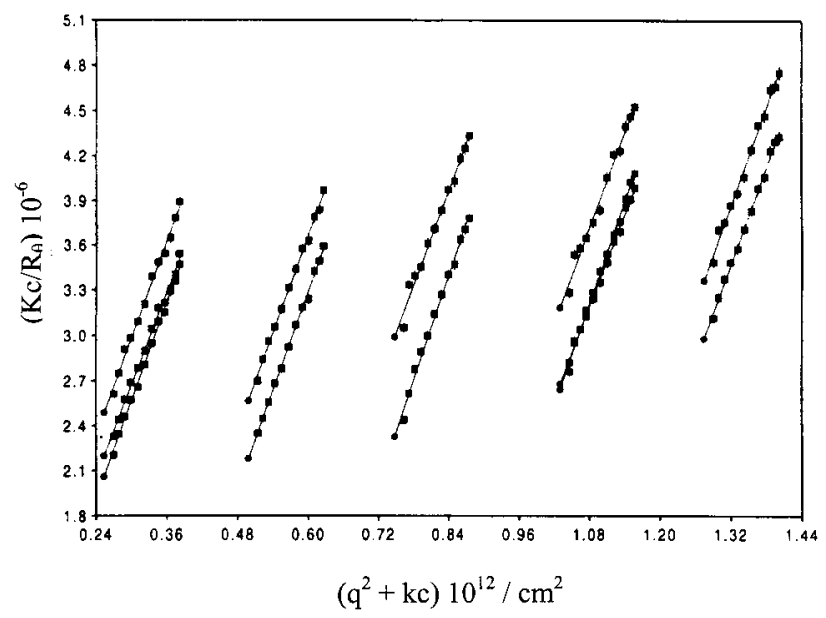

Figure 4. "Floating check". Freshly prepared and immediately after dissolution (within at most $6 \mathrm{~h}$ ) measured solutions of cellulose in cuoxam show no change in the scattering behavior when centrifuged for 20 and $40 \mathrm{~min}$ (the two lower sets of curves). The same solutions centrifuged again $6 \mathrm{~h}$ after the performed measurements display a shift to larger values of the reciprocal scattering intensities (upper set of curves). Apparently a certain amount of the cellulose was now removed.
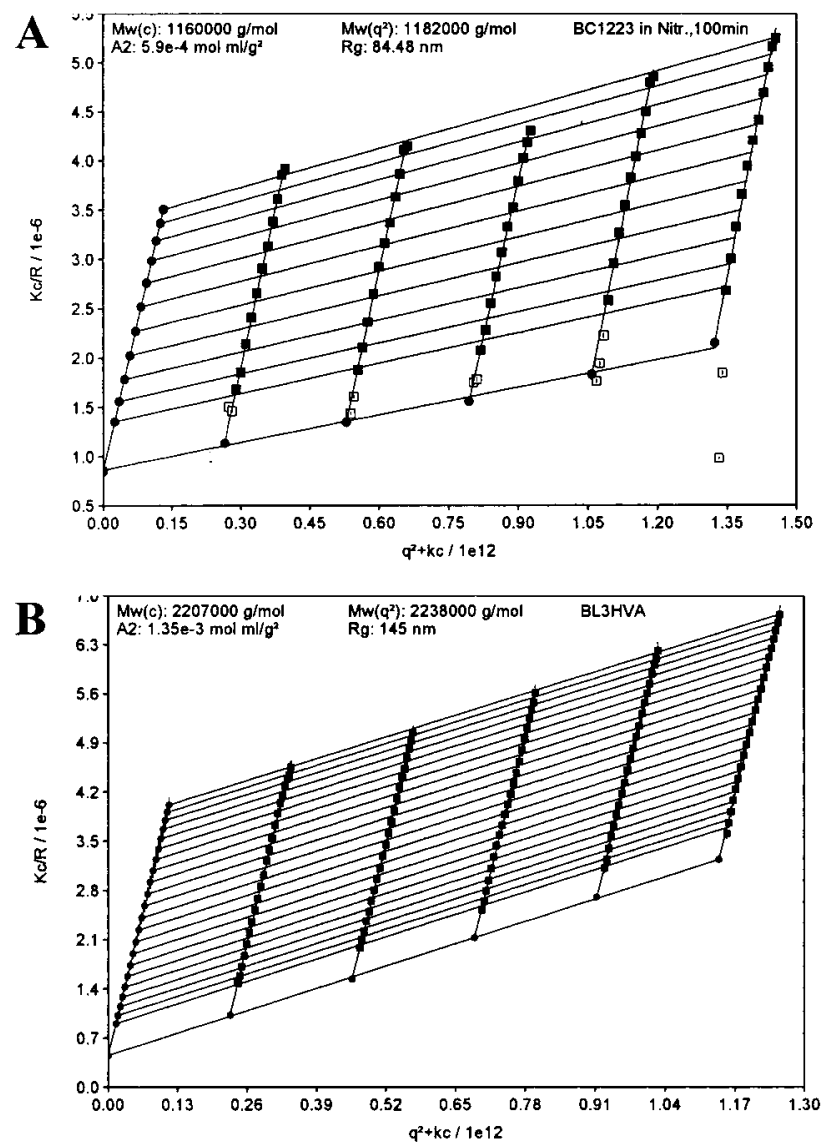

Figure 5. Typical Zimm plots for cellulose: (A) BC 122.3 in $\mathrm{Ni}$-tren, $M_{\mathrm{w}}=1.18 \times 10^{6} \mathrm{~g} / \mathrm{mol}, \mathrm{R}_{\mathrm{g}}=84 \mathrm{~nm}$; (B) Lin3 in Cdtren, $M_{\mathrm{w}}=2.24 \times 10^{6} \mathrm{~g} / \mathrm{mol}, R_{\mathrm{g}}=145 \mathrm{~nm}$.

the solvent which is $\lambda=\lambda_{0} / \mathrm{n}_{0}$. The solvent viscosity enters the Stokes-Einstein relationship

$$
\mathrm{R}_{\mathrm{h}}=\frac{\mathrm{kT}}{6 \pi \eta_{0} \mathrm{D}}
$$

according to which the hydrodynamic radius $R_{h}$ of the macromolecules can be derived from the translational diffusion 


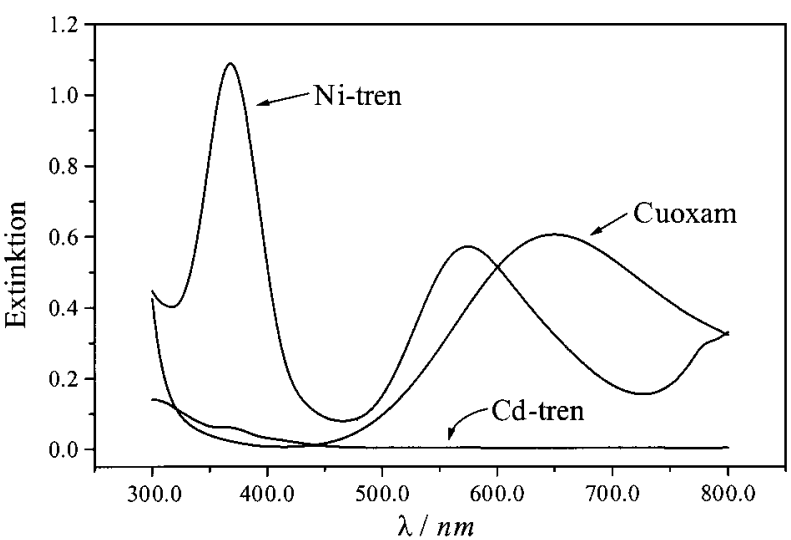

Figure 6. Absorption spectra of $\mathrm{Ni}$-tren, cuoxam, and $\mathrm{Cd}$ tren. Light scattering measurements were made at $\lambda_{0}=457.9$ $\mathrm{nm}$ for $\mathrm{Ni}$-tren and $\lambda_{0}=488 \mathrm{~nm}$ for cuoxam and Cd-tren. The correction factors were $\mathrm{f}_{e}=3.77$ for $\mathrm{Ni}$-tren and $\mathrm{f}_{\mathrm{e}}=1.47$ for cuoxam, by which the scattering intesity had to be multiplied. No correction was necessary for Cd-tren.

coefficient $D_{\text {trans, }}$ which is measured in dynamic LS. As al ready mentioned, the refractive index increments were obtained from a linear relationship of $\mathrm{dn} / \mathrm{dc}$ as a function of $\mathrm{n}_{0}$. Linear regression of literature data gave the relationship (see F igure 3)

$$
\mathrm{dn} / \mathrm{dc}=1.468-0.924 \mathrm{n}_{0}
$$

It may be repeated here that no difference in $\mathrm{dn} / \mathrm{dc}$ was observed for amylose and cellulose tricaranilates. We see no reason to expect differences also for amylose and cellulose in the metal-based solvents. ${ }^{9}$ F urthermore, eq 4 does not contain the influence of the coordinative binding of the metal compounds. The viscosity of the solvent was obtained by measuring the kinematic viscosity in an Oswald viscometer that was corrected by the solvent density for the correct viscosity.

Absorption Correction. In colored solvents the scattering intensity is often remarkably reduced. This reduction of intensity could be accounted for by measuring the extinction $E$ of the solvent and applying Lambert-Beer's law in which the actual diameter of the LS cell $(\mathrm{d}=0.8 \mathrm{~cm})$ was inserted. Figure 6 shows the absorption spectra for the three metal complexing solvents. The selected blue line of the argon laser had the wavelength $\lambda_{0}=457.9 \mathrm{~nm}$, which is close to the minima for cuoxam $\left(\lambda_{0} \min =410 \mathrm{~nm}\right)$ and $\mathrm{Ni}$-tren $\left(\lambda_{0 \mathrm{~min}}=465\right.$ $\mathrm{nm}$ ) absorption curves. F or the Cd-tren sol utions the stronger line of $\lambda_{0}=488 \mathrm{~nm}$ was chosen. The correction factors were $\mathrm{f}$ $\approx 1.47$ for cuoxam and $\mathrm{f} \approx 3.77$ for $\mathrm{Ni}$-tren. No correction was necessary for Cd-tren.

Static Light Scattering. Measurements in Cd-tren were made with a modified SOFICA instrument (design: G. Baur Apparatebau, Hausach, Germany). The instrument is fully computer driven and is based on a new concept for the detection electronics with improved accuracy. The blue line $\left(\lambda_{0}=488 \mathrm{~nm}\right.$ ) of a $10 \mathrm{~mW}$ argon laser (Uniphase, Eching, Germany) was used, and the angular dependence of the scattering intensity was measured in steps of $5^{\circ}$ in a range from $30^{\circ}$ to $145^{\circ}$. On-line software programs for Zimm, Berry, and Guinier plots facilitated the evaluation of molar mas $M_{w_{1}}$ radius of gyration $R_{g}$, and second virial coefficient $A_{2}$. The static LS measurements in cuoxam and $\mathrm{Ni}$-tren were made with the equipment for dynamic LS as described in the next paragraph. The change of instruments was necessary because the required wavelength of $\lambda_{0}=457.9 \mathrm{~nm}$ could only be chosen with the $3 \mathrm{~W}$ argon ion laser (Spectra Physics, Darmstadt, Germany).

Dynamic Light Scattering. An ALV goniometer (ALVVertrieb, Langen, Germany) was used that was equipped with a $3 \mathrm{~W}$ argon ion laser (Spectra Physics). Time correlation functions were measured with an ALV 5000 correlator covering a time domain from $10^{-6}$ to $10^{4} \mathrm{~s}$ in one run. Measurements were made in steps of $10^{\circ}$ in the range from $30^{\circ}$ to $140^{\circ}$. Evaluation by cumulant fits was made only for the cuoxam solutions. In Cd-tren and $\mathrm{Ni}$-tren remaining colloid particles disturbed this analysis. In these cases the analysis was made via Kohlrausch-Williams-Watts stretched exponential curves. ${ }^{24}$ By this technique the relaxation times for each mode could be determined, and the required first cumulant (that no longer contained the contribution of the solvents colloids) was then reconstructed.

\section{Theory}

Static and dynamic light scattering measurements are common techniques in polymer science. Therefore, the following relationships are given without special comments. For detailed information textbooks ${ }^{25-27}$ may be consulted.

Evaluation of static LS data is commonly made on the basis of the Debye equation

$$
\frac{\mathrm{KC}}{\mathrm{R}_{\theta}}=\frac{1}{\mathrm{M}_{\mathrm{w}} \mathrm{P}_{\mathrm{z}}(\mathrm{q})}+2 \mathrm{~A}_{2} \mathrm{C}
$$

The contrast factor was al ready given by eq 2 , and $\mathrm{c}$ is the weight concentration in $\mathrm{g} / \mathrm{mL} . \mathrm{P}_{\mathrm{z}}(\mathrm{q})$ is the particle scattering factor that is sensitive to the structure of the molecules in solution. F or small values of $\mathrm{qR}_{\mathrm{g}}$ it is given by the following equation

$$
\frac{1}{P_{z}(q)}=1+\frac{1}{3} q^{2} R_{g}^{2}+\ldots
$$

with

$$
q=\left(\frac{4 \pi n_{0}}{\lambda_{0}}\right) \sin \left(\frac{\theta}{2}\right)
$$

where $\theta$ is the scattering angle. All other quantities have the common meaning. The subscript $z$ denotes the $z$-average. Thus, from the intercept at $c=0$ and $q=0$ the molar mass $\mathrm{M}_{\mathrm{w}}$ is obtained, and from the initial slopes of the limiting curves $\mathrm{c}=0$ and $\mathrm{q}=0$ the radius of gyration $R_{g}$ and the second virial coefficient $A_{2}$, respectively, are found.

In dynamic LS a normalized intensity-time correlation function (TCF)

$$
g_{2}(t, q) \equiv \frac{\langle i(0, q) i(t, q)\rangle}{\langle i(0, q)\rangle^{2}}
$$

is measured, where $\mathrm{i}(\mathrm{t})$ is the scattering intensity at a certain delay time from a reference timet $=0$. In dilute solution this intensity TCF can be expressed in terms of the normalized field TCF using the Siegert ${ }^{25}$ relationship

$$
\mathrm{g}_{2}(\mathrm{t}, \mathrm{q})=1+\beta\left|\mathrm{g}_{1}(\mathrm{t}, \mathrm{q})\right|^{2}
$$

with a coefficient $\beta$ that depends on the quality of coherence. For short delay times $\mathrm{g}_{1}(\mathrm{t}, \mathrm{q})$ is well approximated by an exponential decay

$$
g_{1}(t, q) \approx \exp \left(-q^{2} D_{a p p}(q) t\right)
$$

with

$$
\mathrm{D}_{\text {app }}(\mathrm{c}, \mathrm{q} \rightarrow 0) \equiv \mathrm{D}_{\mathrm{c}} \approx \mathrm{D}_{\text {trans }}\left(1+\mathrm{k}_{\mathrm{D}} \mathrm{c}\right)
$$

where $k_{D}$ is a coefficient that describes the concentration 
Table 2. Origin of Investigated Cellulose Samples ${ }^{a}$

\begin{tabular}{|c|c|c|c|}
\hline sample & origin of cellulose & $\mathrm{DP}_{\eta}$ & water content (\%) \\
\hline Lin0 & purified cotton linters & 800 & 4.84 \\
\hline Lin1 & buckey linters 4403/020 & 1470 & 5.00 \\
\hline Lin2 & buckey linters $4410 / 030$ & 2000 & 5.61 \\
\hline Lin3 & buckey linters (Memphis) & 7000 & 1.02 \\
\hline Avicel & microcrystalline cellulose $\mathrm{PH}-101$, Fluka & 280 & 5.06 \\
\hline BC122.2 & bacterial cellusoe (laboratory of J ena) & & ca. 5 \\
\hline $\mathrm{BC} 122.3$ & bacterial cellulose (laboratory of J ena) & & ca. 5 \\
\hline BC125.1 & bacterial cellulose (laboratory of J ena) & & ca. 5 \\
\hline $\mathrm{BC} 125.3$ & bacterial cellulose (laboratory of J ena) & & ca. 5 \\
\hline $\mathrm{BC} 125.4$ & bacterial cellulose (laboratory of J ena) & & ca. 5 \\
\hline BC125.5 & bacterial cellulose (laboratory of J ena) & & ca. 5 \\
\hline $\mathrm{BCkr} 2$ & bacterial cellulose (laboratory of J ena) & & ca. 5 \\
\hline Mcell & pulp cellulose MODO, Rhone-Poulenc Rhodia & 570 & 8.32 \\
\hline Ecell & eucalyptus-pulp cellulose & & 6.54 \\
\hline Cell1 & buckey prehydrolyzed sulfate-pulp cellulose V-60 & 800 & 2.34 \\
\hline Cell2 & buckey prehydrolyzed sulfate-pulp cellulose V- 6 & 2000 & 1.25 \\
\hline Cello & borregarde sulfit-pulp cellulose & 800 & 1.24 \\
\hline RCell1 & rayaceta (irradiated pulp cellulose, 100kGy) & 350 & 5.98 \\
\hline RCell2 & rayaceta (irradiated pulp cellulose, 60 kGy) & 430 & 5.73 \\
\hline RCell3 & rayaceta (irradiated pulp cellulose, 40 kGy) & 450 & 5.76 \\
\hline
\end{tabular}

a The $\mathrm{DP}_{\eta}$, determined from viscosity in cuen, was provided by the companies. The water content was determined in our laboratory.

Table 3. Molar Masses (in kg/mol) and Corresponding Degrees of Polymerization of Cellulose Determined from Solutions in Cd-tren, Ni-tren, and Cuoxam with the Optical Parameters of Table $1^{a}$

\begin{tabular}{|c|c|c|c|c|c|c|c|c|}
\hline \multirow[b]{2}{*}{ sample } & \multicolumn{2}{|c|}{ via CTC } & \multicolumn{2}{|c|}{ Cd-tren } & \multicolumn{2}{|c|}{ Ni-tren } & \multicolumn{2}{|c|}{ cuoxam } \\
\hline & $\mathrm{DP}_{\mathrm{w}}$ & $M_{w}$ & $M_{w}$ & $\mathrm{DP}_{\mathrm{w}}$ & $M_{w}$ & $\overline{D P_{w}}$ & $M_{w}$ & $\mathrm{DP}_{\mathrm{w}}$ \\
\hline Lin0 & 900 & 145.9 & 283 & 1188 & 274.9 & 1256 & 257 & 978 \\
\hline Lin1 & & & 337 & 1415 & 300.4 & 1372 & 301 & 1146 \\
\hline Lin2 & & & 491 & 2062 & 473 & 2176 & 514 & 1957 \\
\hline Lin3 & & & 2290 & 10035 & 1710 & 7811 & n.s. & n.s. \\
\hline Avicel & & & 56.0 & 235 & 52.6 & 240 & 54.3 & 207 \\
\hline BC 122.2 & 5107 & 828 & 1318 & 5534 & 1200 & 5481 & 1360 & 5178 \\
\hline BC122.3 & 6267 & 1200 & 1502 & 6383 & 1160 & 5299 & 1460 & 5558 \\
\hline BC125.1 & 5383 & 872.8 & 1070 & 4493 & 1001 & 4573 & n.s. & n.s. \\
\hline BC125.3 & 8111 & 1320 & 1690 & 7091 & n.s. & n.s. & n.s. & n.s. \\
\hline BC 125.4 & $5258^{c}$ & 862.6 & 2371 & 9956 & n.s. & n.s. & n.s. & n.s. \\
\hline BC 125.5 & 5651 & 916.2 & 1361 & 5715 & n.s. & n.s. & n.s. & n.s. \\
\hline $\mathrm{BC} k \mathrm{r}$ & & & 807 & 3386 & & & & \\
\hline Mcel & & & 214 & 898 & & & & \\
\hline ECel & & & 320 & 1344 & 291.7 & 1333 & 318 & 1211 \\
\hline Cel0 & & & 420 & 1763 & 376.1 & 1718 & 398 & 1515 \\
\hline Cel 1 & & & 256 & 1075 & 252.9 & 1155 & 270 & 1028 \\
\hline Cel2 & & & 466 & 1957 & 431.7 & 1972 & 452 & 1721 \\
\hline Rcel 1 & & & 136 & 571 & 132.7 & 606 & 145 & 552 \\
\hline Rcel2 & & & 178 & 747 & 175.1 & 800 & 212 & 807 \\
\hline Rcel3 & & & 218 & 915 & 193.5 & 884 & 235 & 895 \\
\hline
\end{tabular}

a From a linters sample and five bacterial celluloses the cellulose tricabanilate (CTC) derivatives were prepared and measured by LS in dioxan. The corresponding degree of polymerization was determined and the corresponding cellulose molar mass derived. These data served as basis for the determination of the correction factor $\mathrm{f}$ listed in Table 1 ; for details see text. ${ }^{\mathrm{b}} \mathrm{n} . \mathrm{s}$. $=$ sample was not completely soluble. ' This value is evidently too low; it was not included in the calibration.

dependence. A double extrapolation of $D_{a p p}(c, q)$ has to be performed to get the translational diffusion coefficient required in eq 3 for the derivation of the hydrodynamic radius $R_{h}$.

\section{Results}

Samples. Three cellulose types were characterized by static and dynamic light scattering and by capillary viscometry in the dilute regime. The first group consisted of four native cotton linters samples, $\mathrm{DP}_{\mathrm{w}}=970$ 9300, and a highly degraded microcrystalline cellul ose (Avicel $\mathrm{DP}_{\mathrm{w}}=280$ ). The second group contained bacterial celluloses synthesized in J ena by Acetobacter $x y-$ linium. ${ }^{25}$ The degree of polymerization could be varied in a range of $\mathrm{DP}_{\mathrm{w}}=3300-9700$ by changing nutrition conditions. The last group consisted of commercially available pulp celluloses. Details of the cellul ose origin are given in Table 2.
The molar masses and degrees of polymerization in the three metal-based solvents are given in Table 3 and are compared with the data of some cellulose tricarbanilates (CTC). The molar masses in the metal-based solvents are in all cases larger than derived from the CTC samples, which reflects the effect of coordinative binding of the complex to the cellul ose chain. The ratio of the molar masses gives the correction factors of Table 1. The factors of Table 1 were obtained as follows. We first determined the ratio of molar masses for the five samples measured in Cd-tren. In a second step the average factor between $\mathrm{Ni}$-tren and $\mathrm{Cd}$-tren and between cuoxam and Cd-tren were determined and multiplied by the factor for Cd-tren. All data of the following results refer to mol ar masses corrected by these factors. The values of the correction factors are all larger than unity and can be considered as a measure of coordinative binding strength. 
Table 4. Molar Mass $M_{w}(\mathrm{~kg} / \mathrm{mol})$, Radius of Gyration $\mathbf{R}_{\mathbf{g}}(\mathrm{nm})$, Hydrodynamic Radius $\mathbf{R}_{\mathrm{h}}$ (nm), and Second Virial Coefficient $A_{2}$ (mol $\mathrm{mL} / \mathrm{g}^{2}$ ) of Cellulose in Cd-tren, Ni-tren, and Cuoxama

\begin{tabular}{|c|c|c|c|c|c|c|c|c|c|c|c|c|}
\hline \multirow[b]{2}{*}{ sample } & \multicolumn{4}{|c|}{ Cd-tren } & \multicolumn{4}{|c|}{$\mathrm{Ni}$-tren } & \multicolumn{4}{|c|}{ cuoxam } \\
\hline & $\mathrm{M}_{\mathrm{w}}$ & $\mathrm{R}_{\mathrm{g}}$ & $\mathrm{R}_{\mathrm{h}}$ & $10^{4} \mathrm{~A}_{2}$ & $M_{w}$ & $\mathrm{R}_{\mathrm{g}}$ & $\mathrm{R}_{\mathrm{h}}$ & $10^{4} \mathrm{~A}_{2}$ & $M_{w}$ & $\mathrm{R}_{\mathrm{g}}$ & $\mathrm{R}_{\mathrm{h}}$ & $10^{4} \mathrm{~A}_{2}$ \\
\hline $\operatorname{Lin} 0$ & 283 & 41 & 23 & 15.1 & 275 & 42 & 19 & 11.6 & 257 & 41 & 17 & 8.5 \\
\hline Lin1 & 337 & 48 & 27 & 15.5 & 300 & 39 & 15 & 93 & 301 & 43 & 18 & 8.9 \\
\hline $\operatorname{Lin} 2$ & 492 & 54 & 31 & 13.4 & 476 & 53 & 19 & 10.7 & 514 & 57 & 25 & 11.0 \\
\hline Lin3 & 2390 & 134 & 78 & 11.6 & 1711 & 101 & 44 & 6.3 & & & & \\
\hline Avicel & 56.0 & 21 & 11 & 16.0 & 52.6 & 20 & 8 & 13.3 & 54.3 & 19 & 7 & 13.9 \\
\hline BC 122.2 & 1318 & 91 & 66 & 9.5 & 1124 & 84 & 29 & 5.9 & 1360 & 88 & 38 & 7.6 \\
\hline $\mathrm{BC} 122.3$ & 1502 & 98 & 68 & 9.7 & 1160 & 85 & 33 & 5.9 & 1462 & 103 & 44 & 9.9 \\
\hline BC125.1 & 1070 & 85 & 54 & 10.3 & 1001 & 76 & 32 & 6.0 & n.s. & n.s. & n.s. & n.s. \\
\hline BC125.3 & 2070 & 124 & 66 & 10.6 & n.s. & n.s. & n.s. & n.s. & n.s. & n.s. & n.s. & n.s. \\
\hline ВС125.4 & 2371 & 127 & 70 & 10.9 & n.s. & n.s. & n.s. & n.s. & n.s. & n.s. & n.s. & n.s. \\
\hline BC 125.5 & 1631 & 106 & 58 & 8.5 & n.s. & n.s. & n.s. & n.s. & n.s. & n.s. & n.s. & n.s. \\
\hline CelO & 421 & 59 & 37 & 12.6 & 376 & 55 & 21 & 9.5 & 398 & 57 & 23 & 11.3 \\
\hline Cel1 & 257 & 43 & 24 & 13.5 & 253 & 46 & 17 & 12.2 & 270 & 39 & 18 & 10.0 \\
\hline Cel2 & 465 & 60 & 35 & 13.8 & 432 & 56 & 21 & 11.0 & 452 & 56 & 24 & 8.6 \\
\hline Mcel & 213 & 40 & 27 & 10.1 & & & & & & & & \\
\hline ECel & 320 & 50 & & 11.1 & 292 & 45 & 18 & 8.5 & 318 & 47 & 19 & 13.4 \\
\hline RCel1 & 136 & 40 & 21 & 15.1 & & & & & & & & \\
\hline RCel2 & 178 & 42 & 23 & 13.2 & & & & & & & & \\
\hline RCel3 & 219 & 45 & 28 & 10.5 & & & & & & & & \\
\hline
\end{tabular}

a Note: the molar masses were not corrected with the calibration factors $f$ as given by Table 1 .

Dimensions of Cellulose Chains. Evaluations of static LS intensities were made from common Zimm plots or Berry plots. The corresponding dynamic LS measurements were obtained from dynamic Zimm plots, which are similar to the static Zimm plots but now $D_{a p p^{-}}$ $(\mathrm{q}, \mathrm{c})$, instead of $\mathrm{Kc} / \mathrm{R}_{\theta}$, is plotted against $\mathrm{q}^{2}+\mathrm{kc}$. As was shown in previous papers, ${ }^{29}$ the combination of static with dynamic Zimm plots gives in favorite cases six parameters, which are $M_{w}, R_{g}, A_{2}$ (static LS), $D_{\text {trans }}$ or $R_{h}$, a coefficient $C$, and another coefficient $k_{D}$ (dynamic LS), where the parameters in the two sets correspond to the intercept, initial slope of the angular dependence, and initial slope in the concentration dependence, respectively. The coefficient $k_{D}$ was al ready defined in eq 11; it depends on thermodynamic and hydrodynamic interactions among the particles and is sometime called a dynamic virial coefficient. The coefficient $\mathrm{C}$ is defined by the following relationship: ${ }^{27}$

$$
D_{a p p}(q, c)=D_{c}\left(1+C q^{2} R_{g}{ }^{2}\right)
$$

It contains the influence of the longest internal mode of segmental motion. ${ }^{30,31}$

All molecular parameters depend on the molar mass (see Table 4). Figure 7 shows the result for $R_{g}$ and $R_{h}$ in $\mathrm{Cd}$-tren and $\mathrm{Ni}$-tren. The filled symbols refer to native celluloses (cotton linters and bacterial celluloses) and the open symbols to pulp celluloses. The results from the two solvents look very similar to each other, but the ratio of the radius of gyration to the hydrodynamic radius is larger in $\mathrm{Ni}$-tren than in Cd-tren. Furthermore, the data from pulp celluloses are in every case larger than those from native celluloses. This may have two reasons. It can mean either that the extraction procedure from wood caused a slight but significant chemical modification, for instance, by introducing electrolytic charges to the chain, or that the accompanying hemicelluloses were not sufficiently well removed. In both cases a chain stretching is to be expected via Coulomb repulsion in polyelectrolytes or via side-by-side adsorption of hemicellulose chains.

The results of $R_{g}$ from the cuoxam solutions are plotted in Figure 8 where the data from freshly prepared samples (filled symbols) are compared with those previ-
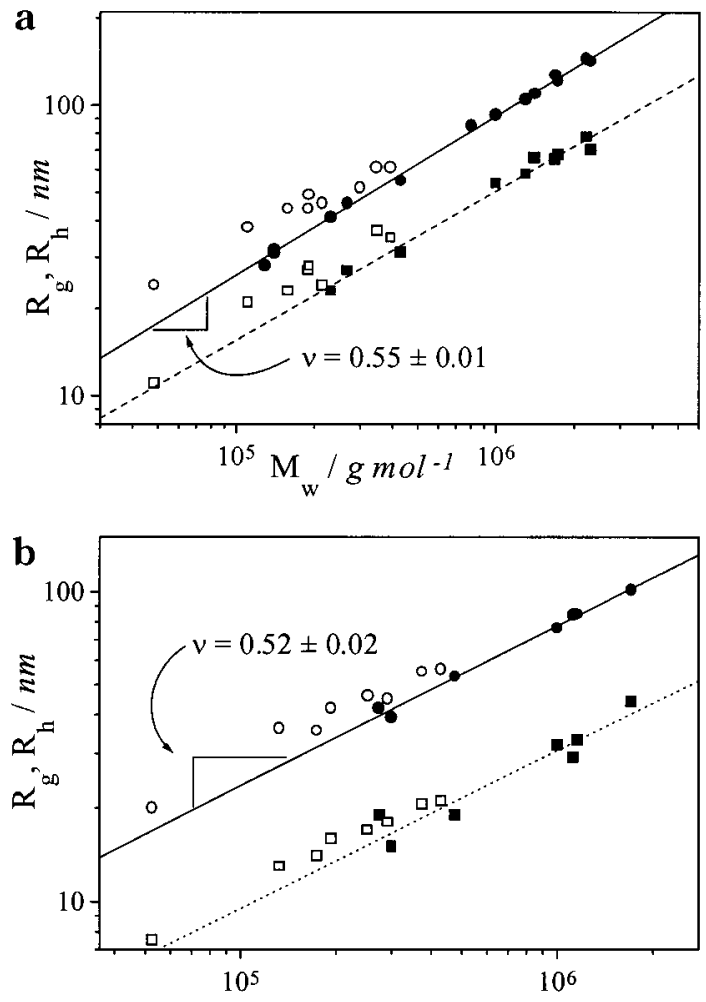

Figure 7. Molar mass dependencies of the radius of gyration $\mathrm{R}_{\mathrm{g}}$ (upper curves) and hydrodynamic radius $\mathrm{R}_{\mathrm{h}}$ (lower curves) for cellulose (a) in Cd-tren and (b) in Ni-tren. Filled symbols: native and bacterial celluloses; open symbols: pulp celluloses of different origin.

ously published by Seger et al.8,32 Searching for reasons of the deviations, we noticed that in the previous study solutions were prepared the evening before measurement. Repeat of this procedure in the present study disclosed that aging occurred. New solvent aggregates were formed, and these were strongly coupled to the cellulose chain. This conclusion was drawn from the fact that an appreciable amount of cellulose was now removed by centrifugation which did not occur with freshly prepared solutions and immediate measurement after dissolution. However, the preparation of cellulose 

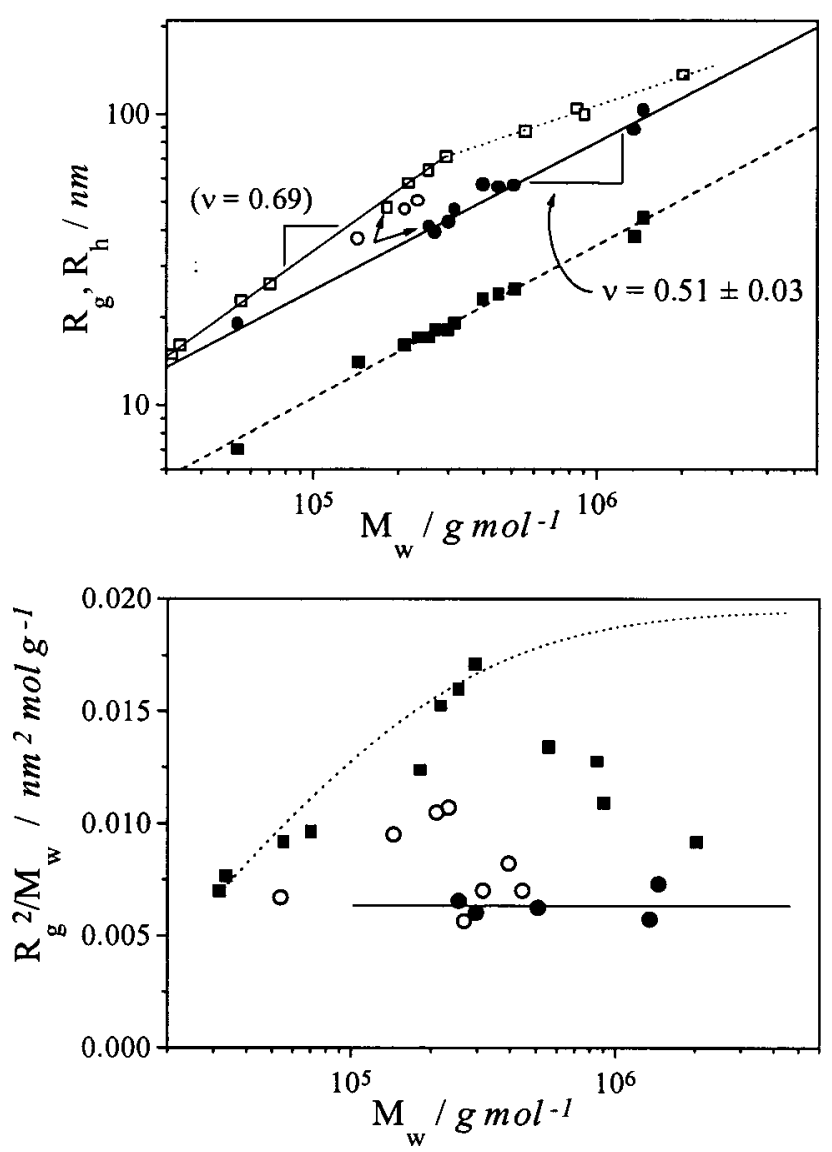

Figure 8. Top: $R_{g}$ (filled circles) and $R_{h}$ (filled squares) as a function of $M_{w}$ for cellulose in cuoxam (this sudy). No differentiation of the various cellulose types was made. The open squares correspond to earlier measurements ${ }^{8}$ where the solutions were prepared $12 \mathrm{~h}$ before measurement. Because of aggregation the concentration was decreased and resulted in apparent molar masses that are too large (see text). Bottom: ratio $R_{g}^{2} / M_{w}$ as a function of $M_{w}$. Filled symbols correspond to native and open symbols to pulp celluloses.

solutions in cuoxam is delicate, and slight changes in the procedure may result in different properties.

The curves in Figures 7 and 8 can be well described by power laws, for instance

$$
\mathrm{R}_{\mathrm{g}}=\mathrm{K}_{\mathrm{R}_{\mathrm{g}}} \mathrm{M}_{\mathrm{w}}{ }^{v} \mathrm{~g}
$$

and a similar relationship for $R_{h}$. The exponents are given in Table 5 together with the other exponents for the second virial coefficient and the intrinsic viscosity.

Intrinsic Viscosity. The intrinsic viscosity is usually determined by linear extrapolation of the reduced viscosity against zero concentration according to the relationship

$$
\frac{\eta_{\mathrm{sp}}}{\mathrm{c}}=[\eta]\left(1+\mathrm{k}_{\mathrm{H}}[\eta] \mathrm{c}+\ldots\right)
$$

neglecting higher terms in concentration, where $\mathrm{K}_{\mathrm{H}}$ is Huggins' constant. The high molar mass celluloses, however, exhibited a significant quadratic term. Such behavior seems to be typical for cellulose derivatives and was al ready observed by Schulz ${ }^{33}$ many years ago. He suggested a plot of $\eta_{\text {sp }} / \mathrm{c}$ against $\eta_{\text {sp. }}$. This plot, known as the Schulz-Blaschke plot, ${ }^{33}$ gave a linearization of the concentration dependence. Figure 9 shows that such a linearization was also obtained with the present
Table 5. Experimental Molecular Parameters Compared with Theoretical Predictiona

\begin{tabular}{llll}
\hline & \multicolumn{1}{c}{ Cd-tren } & \multicolumn{1}{c}{ Ni-tren } & \multicolumn{1}{c}{ cuoxam } \\
\hline$v_{\text {exp }}$ & $0.55 \pm 0.01$ & $0.52 \pm 0.02$ & $0.51 \pm 0.03$ \\
$\mathrm{~K}_{\mathrm{R}_{g}}\left(\mathrm{~nm} \mathrm{~mol}^{\nu} / \mathrm{g}^{\nu}\right)$ & $0.047 \pm 0.007$ & $0.062 \pm 0.019$ & $0.071 \pm 0.030$ \\
$\rho=\mathrm{R}_{\mathrm{g}} / \mathrm{R}_{\mathrm{h}}$ & $1.84 \pm 0.14$ & $2.59 \pm 0.19$ & $2.51 \pm 0.24$ \\
$\mathrm{a}_{\eta}$ & $0.72 \pm 0.03$ & & $0.81 \pm 0.03$ \\
$v_{\text {scal }, \eta}$ & $0.57 \pm 0.01$ & & $0.61 \pm 0.02$ \\
$\mathrm{~K}_{\eta}(\mathrm{mL} / \mathrm{g})$ & $2.64 \pm 0.56$ & & $0.77 \pm 0.03$ \\
$\mathrm{~A}_{\mathrm{A}_{2}}$ & $-0.12 \pm 0.03$ & $-0.28 \pm 0.04$ & $-0.09 \pm 0.06$ \\
$v_{\text {scal }, \mathrm{A}_{2}}$ & $0.63 \pm 0.01$ & $0.57 \pm 0.01$ & $0.64 \pm 0.02$ \\
$\mathrm{I}_{\mathrm{K}, \text { Benoit-Doty }}(\mathrm{nm})$ & $15.8 \pm 1.4$ & $10.2 \pm 0.8$ & $13.1 \pm 1.2$ \\
$\mathrm{I}_{\mathrm{K} \text { Koyama }}(\mathrm{nm})$ & $14.8 \pm 1.0$ & $10.5 \pm 0.8$ & $12.7 \pm 1.9$ \\
$\mathrm{C}_{\propto}$ & $24.7 \pm 2.7$ & $20.1 \pm 1.6$ & $25.0 \pm 3.0$
\end{tabular}

a The exponents and amplitude factors were derived from eq 13 with similar relationships for $\mathrm{A}_{2}$ and $[\eta]$ where $v_{\mathrm{g}}, \mathrm{a}_{\mathrm{A}_{2}}$, and $\mathrm{a}_{\eta}$ are the exponents in the molar mass dependencies of $R_{g}$ and $R_{h}$, the second viral coefficient and the intrinsic viscosity, respectively. The theoretical $v_{\text {scal,j }}$ were derived via scaling relationships $v_{\text {scal, }}$ $=\left(\mathrm{a}_{\mathrm{A}_{2}}+2\right) / 3$ and $v_{\mathrm{scal}, \eta}=\left(\mathrm{a}_{\eta}+1\right) / 3$, respectively. Kuhn segment lengths $I_{K}$ were obtained from the molar mass dependence of $R_{g}$ according to the Benoit-Doty relationship eq 18a for polydisperse chains with $M_{w} / M_{n}=2$ and from the angular dependence of scattered light using the Koyama theory of wormlike chains. The characteristic ratio was obtained from the approximation $\mathrm{C}_{\propto}=$ $\mathrm{I}_{\mathrm{K}} / \mathrm{I}$, with the bond length $\mathrm{I}=\mathrm{O}_{1}-\mathrm{O}_{4}{ }^{\prime}$ distance.

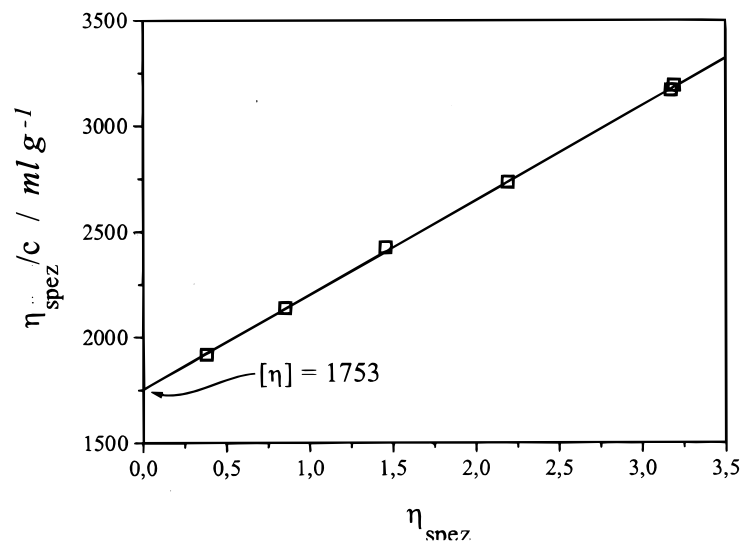

Figure 9. Dependence of the reduced viscosity $\eta_{\text {sped }} / \mathrm{c}$ on the specific viscosity $\eta_{\text {spec }}$ which increases with the concentration (Schulz-Blaschke ${ }^{33}$ plot). The corresponding plot against concentration gave a strong upturn that is linearized by the Schulz-Blaschke procedure.

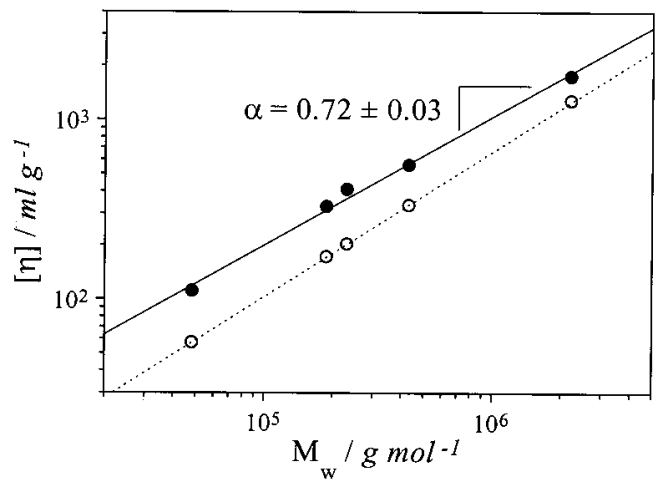

Figure 10. Molar mass dependence of the intrinsic viscosity for cellulose in cuoxam (open circles) and Cd-tren (filled circles).

celluloses and allowed reliable extrapolation to zero concentration. The molar mass dependence of the intrinsic viscosities in Cd-tren and cuoxam is shown in Figure 10. The data are given in Table 4, and the constants and exponents of the power law behavior are listed in Table 5 together with some other molecular 

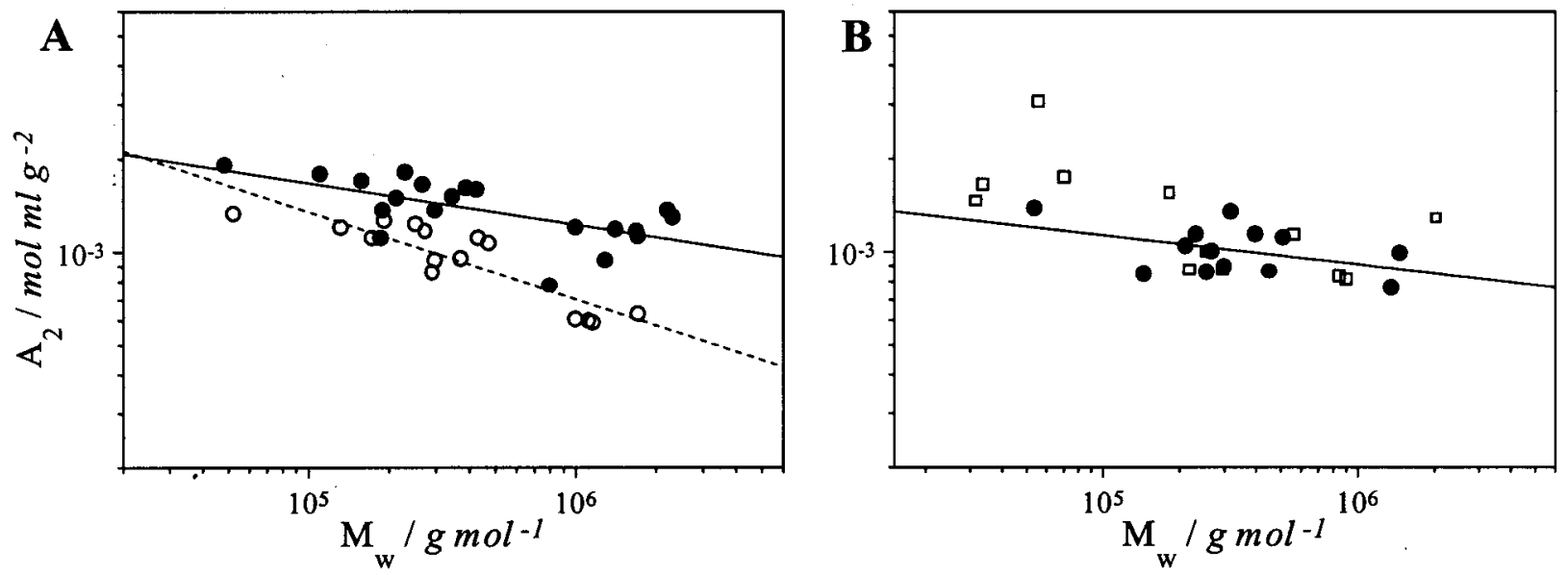

Figure 11. Molar mass dependencies of the second virial coefficients (A) in Cd-tren (filled circles) and $\mathrm{Ni}$-tren (open circles) and (B) in cuoxam (filled circles: this study; open squares: results from ref 8).

parameters. Again common behavior of linear chains is observed.

Second Virial Coefficient. Surprisingly high second virial coefficients were found (Table 4). Figure 11 exhibits the molar mass dependencies in the three cellulose solvents. No differentiation between the three types of cellulose was made. The exponents in the power law dependencies are small, as could be expected for molecularly dispersed linear chains, but vary slightly in value. The reason for this behavior may be found in a certain chain stiffness but could also be seen in marginally good solvent behavior. M ostly the exponents for the various quantities are rel ated to each other. The corresponding scaling relationship for the exponent $v$ of the radius of gyration and that for the second virial coefficient is given by ${ }^{34}$

$$
v_{\mathrm{g}}=\frac{\mathrm{a}_{\mathrm{A}_{2}}+2}{3}
$$

and for the viscosity one has

$$
v_{\mathrm{g}}=\frac{\mathrm{a}_{\eta}+1}{3}
$$

In Table 5 the experimental $v_{\text {exp }}$ exponents are compared with those obtained via eqs $15 \mathrm{a}$ and $15 \mathrm{~b}$. The theoretical value of the renormalization group theory $(R G)$ in the good solvent limit is $\nu_{R G}=0.588 .{ }^{35} \mathrm{Larger}$ exponents give evidence for chain stiffness, and smaller ones indicate marginal solution behavior. One notices that the scaling relationships are not well obeyed; in all cases the calculated data for $v$ are somewhat larger than the directly measured ones. The effect is most devel oped for cuoxam.

\section{Discussion}

The present results clearly demonstrate common solution behavior of molecularly dispersed linear chains in the three metal-based solvents $\mathrm{Cd}$-tren, $\mathrm{Ni}$-tren, and cuoxam. Thus, the predictions, made on the basis of crystalline structures of monosaccharides and the study of stability constants of the complexes, are evidently correct and valid. ${ }^{6,7,11} \mathrm{Cd}$-tren exhibits the strongest solvating power and could dissolve even the highest degrees of polymerization of bacterial celluloses with $\mathrm{DP}_{\mathrm{w}}=9700$. Ni-tren has lower efficiency and does not dissolve chains of $\mathrm{DP}_{\mathrm{w}}>5500$, and cuoxam seems to be the weakest solvent. This order corresponds to the values of the $v$ exponents. However, the order derived from the exponents of the second virial coefficients is different and, and similar ranging is obtained for the intrinsic viscosity. As al ready mentioned, this could be caused by differences in chain stiffness in these solvents. This effect could be a result of differences in the stability constants for coordinative binding of the metal complexes to the chain. The various effects are considered in detail in the next paragraph.

Chain Stiffness. The rigidity of chains is characterized by two quantities which are the Kuhn segment length $I_{K}$ and the number of Kuhn segments per contour length $\mathrm{N}_{\mathrm{K}}$. In literature often only the Kuhn segment length is given as stiffness measure, but this can be misleading because the Kuhn length depends on the bond length I between two neighbored repeating units. The bond length of the anhydro glucose unit in cellulose is I $=0.515 \mathrm{~nm}$ and that for a typical vinyl polymer only $\mathrm{I}=0.25 \mathrm{~nm}$. Thus, the cellulose stiffness is often overestimated by a factor of 2 . More sensible is the use of the dimensionless ratio

$$
\frac{\mathrm{I}_{\mathrm{K}}}{\mathrm{l}} \approx \mathrm{C}_{\infty}
$$

which to a good approximation is Flory's characteristic ratio and a measure for rotational hindrance of the units around their bonds. ${ }^{36}$

For sufficiently long chains when $\mathrm{qR}_{\mathrm{g}}>2$, the Kuhn segment length and the number of Kuhn segments can be derived from the angular dependence of the scattered light. In the region of large $\mathrm{qR}_{\mathrm{g}}$ values the angular dependence becomes sensitive to the molecular structure. Analysis of the curves becomes possible by application of Koyama's theory ${ }^{37-39}$ for wormlike chains. The rigid-rod properties of the Kuhn segment have to be taken into account, which are effective at very large $q$ values. The rod properties are best recognized in the Casassa-Holtzer pl ot. ${ }^{40,41}$ Here the scattering intensity $\mathrm{R}_{\theta}$ is multiplied by the magnitude of the q-vector which for long rods gives a constant plateau value that no longer depends on the q value

$$
\frac{\mathrm{qR}_{\theta}}{\mathrm{KC}} \rightarrow \pi \mathrm{M}_{\mathrm{L}}=\pi\left(\frac{\mathrm{M}}{\mathrm{L}}\right)
$$

where $\mathrm{M}$ is the molar mass of the rod, $\mathrm{L}$ is the length, 


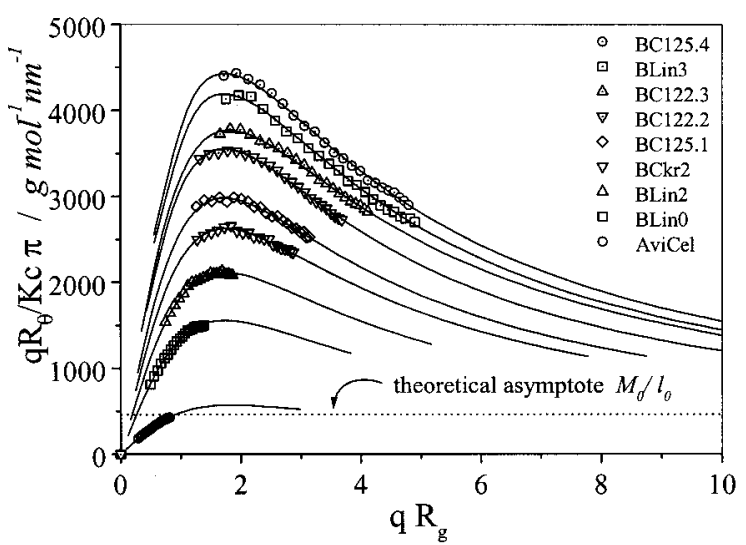

Figure 12. Casassa-Holtzer plots for nine cellul ose samples in Cd-tren. The lines correspond to best fits via the Koyama theory ${ }^{37}$ of wormlike chains. Similar curves were obtained in the other two solvents.

and $M_{L}$ is the linear mass density. The latter can be calculated from the molar mass and the bond length of the repeating unit in cellulose which gives $\mathrm{M}_{\mathrm{L}}=314.56$ $\mathrm{g} /(\mathrm{mol} \mathrm{nm})$. The asymptote is reached from below for rigid rods, but a maximum is passed for semiflexible chains, where the ratio of this maximum height to the plateau value of the rod increases with the number of Kuhn segments and is thus a measure of flexibility. ${ }^{42}$ Figure 12 shows such Casassa-Holtzer plots for nine samples measured in Cd-tren. The full lines correspond to fits by the Koyama theory ${ }^{37}$ using the abovementioned linear mass density. The fits were done with a special program 39 that approached the best solution by an iterative process. Physically reasonable constraints had to be set as initial values. Similar curves were obtained with the other solvents. The reliability of the fits was controlled with the radius of gyration. Benoit and Doty ${ }^{43}$ derived an equation for the radius of gyration for chains of different stiffness (i.e., Kuhn segment length and number of Kuhn segments). This relationship was complemented by Schmidt, ${ }_{4}$ who performed the average for Schulz-Zimm length distributions. We assumed a most probable distribution $\left(M_{w} / M_{n}=2\right)$ for all samples.The radius of gyration now can be expressed by the simple relationship

$$
R_{g}=L_{w}\left[\frac{1}{4\left(1+N_{K}\right)}\right]^{1 / 2}
$$

where

$$
\mathrm{L}_{\mathrm{w}}=\mathrm{IDP}_{\mathrm{w}}=\mathrm{I}_{\mathrm{K}} \mathrm{N}_{\mathrm{K}}
$$

The results of the fits are collected in Table 5. The difference in stiffness is not pronounced; apparently, cellulose has the highest chain stiffness in Cd-tren and the weakest in Ni-tren. This conclusion is now in agreement with order in the exponents $a_{A_{2}}$ for the second virial coefficient.

F ujita ${ }^{45}$ collected data from polymers of very different rigidity and plotted $R_{g}^{2} l_{K}^{2}$ against $N_{K}$ in a doublelogarithmic scale. Healso checked the relationships for influences of excluded volume. Figure 13 shows the result for monodisperse chains and for chains with most probable distribution. Fujita noticed that the excluded volume has an effect only when the chain is longer than $50 N_{k}$. Such an influence should be noticeable for

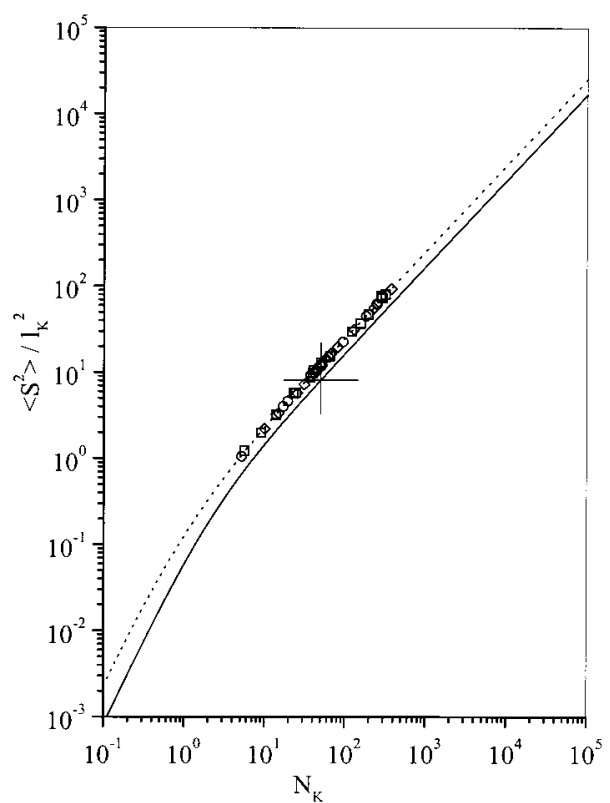

Figure 13. Plot of $\left(R_{g} / I_{K}\right)^{2}$ against the number of Kuhn segments $\mathrm{N}_{\mathrm{K}}$ per chain according to Fijita. ${ }^{45}$ Symbols refer to measurements, full line refers to monodisperse chains, dashed line refers to polydisperse chains with $M_{w} / M_{n}=2$. In good solvents the effect of exduded vol ume becomes noticeable when $\mathrm{N}_{\mathrm{K}}>50$, indicated by the cross. The assumption of polydisperse cellulose chains is well confirmed with the present samples. $I_{K}$ is the Kuhn segment length, and $\left\langle S^{2}\right\rangle=R_{g}{ }^{2}$ is the mean-square radius of gyration.

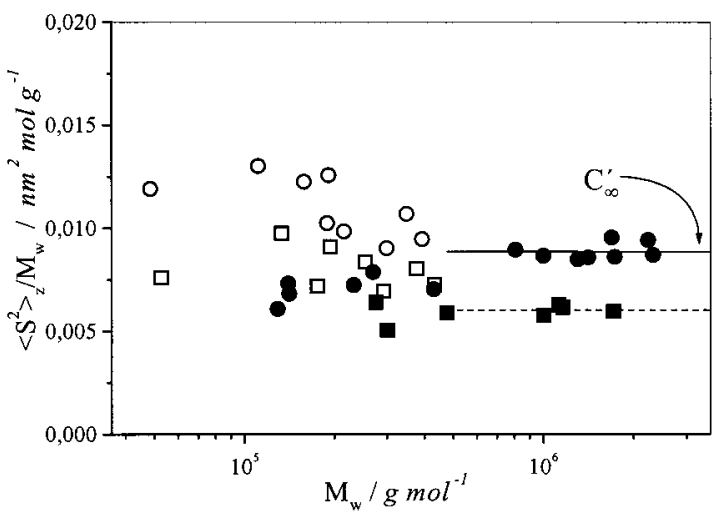

Figure 14. Plot of $\left\langle S^{2}\right\rangle / M_{w}$ against the molar mass $M_{w}(A)$ for cellulose in Cd-tren (circles) and Ni-tren (squares) (B) in cuoxam (circles: this study, squares: ref 8, see text). The filled symbols refer to native and the open to pulp celluloses. No influence of intrachain excluded-volume interaction is recognized. $C_{\propto}{ }^{\prime}=\left(I^{2} / 6 M_{0}\right) C_{\propto}$.

samples with $\mathrm{DP}_{\mathrm{w}}>1600$. Our data follow the relationship for polydisperse chains without excluded-volume effects.

A more sensitive check can be made by considering the ratio $R_{g}^{2} / M_{w}$. A constant plateau should be reached for chains that approach the behavior of Gaussian chains, whereas in typical good solvents the plot should approach an increase with power of $0.176 .35,46$ In all three solvents a Gaussian plateau is reached (Figure 14) which indicates that the systems are not yet in the region of thermodynamically good solvents. The plateau height is proportional to Flory's characteristic ratio at large chain lengths. Apparently $\mathrm{Ni}$-tren is less strongly bound to the cellulose chain than Cd-tren, which is equivalent to lower chain stiffness in $\mathrm{Ni}$-tren than in Cd-tren. 


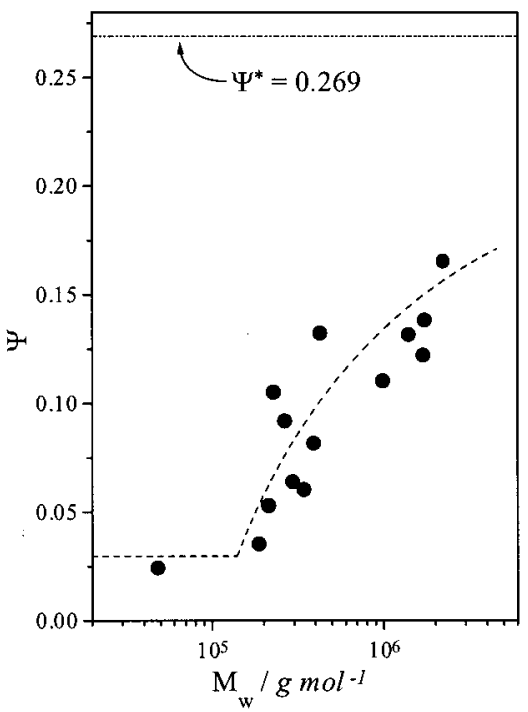

Figure 15. Coil interpenetration function $\Psi(\mathrm{z})$ as a function of the molar mass for cellulose in the three metal-based solvents: (A) Cd-tren, (B) Ni-tren, (C) cuoxam. According to Fujita, ${ }^{42}$ the effect of excluded volume becomes not effective before $N_{K}=50$. The asymptotic limit of $\Psi^{*}=0.269$ is not reached, because $N_{K}$ remained too small even for the longest cellulose chains.

A special comment is required concerning the behavior of cuoxam. With freshly prepared samples again a plateau of almost the same height as for $\mathrm{Ni}$-tren is obtained. In previous papers 8,9 a different behavior was reported according to which the values pass through a maximum, which is larger than the plateau height, and then decreased strongly. As already mentioned, this difference in behavior may be an effect of aging. In our interpretation colloid clusters of the solvent are adsorbed onto the chain. Part of the chains with adsorbed clusters are removed on ultracentrifugation. Now an apparent molar mass is measured that is smaller by the reduced concentration. Adsorption of a colloid cluster can also cause stiffening of the chain. The downturn beyond the maximum was inter preted as intramolecular cross-linking. This view remains valid, but the intramolecular reaction would be caused here not by a single complex but more likely due to coupling via a colloid cluster.

Interpenetration F unction $\boldsymbol{\Psi}$. The second virial coefficient is described in general terms by the equa$\operatorname{tion}^{46}$

$$
A_{2}=4 \pi^{3 / 2} N_{a} \frac{R_{g}{ }^{3}}{M_{w}{ }^{2}} \Psi(z)
$$

with $\mathrm{z}$ an interaction parameter given by ${ }^{46}$

$$
\mathrm{z}=\left(\frac{3}{\left.2 \pi\right|_{\mathrm{K}} ^{2}}\right)^{3 / 2} \beta \mathrm{N}_{\mathrm{K}}^{1 / 2}
$$

in which $\beta$ is the binary cluster integral that describes the excluded vol ume of a Kuhn segment. The function $\Psi(z)$ describes how deep two coi Is of macromolecules can penetrate each other. Under $\Theta$-conditions one has $z=$ 0 and full interpenetration, i.e., $\Psi(z)=0$. With increasing repulsive interactions $z$ and $\Psi(z)$ increase until a constant value $\Psi^{*}=0.269$ is reached. ${ }^{35}$ (Note that $z$ increases proportional to $N_{K}^{1 / 2}$.) Figure 15 shows a plot of $\Psi(\mathrm{z})$ as a function of $\mathrm{M}_{\mathrm{w}}^{1 / 2}$. The curves start at very low values, and only beyond $M_{w}>150000\left(D_{w}>900\right.$, $\mathrm{N}_{\mathrm{K}}>35$ ) does a sharp increase occur. This confirms Fujita's findings that excluded-volume effects become noticeable only for $N_{K}>50$. Thus, the cellulose chains exist in the present solvents in a transition region from marginal to good-solvent behavior, not because of a weak solvation power, but because of the existing chain stiffness and small number of Kuhn segments. In this transition regime scaling relationships are not exactly fulfilled.

Another point is of interest. Because of the chain stiffness the second virial coefficient at low molar mass approaches that of a chain of less than one Kuhn segment in length. If the Kuhn segement is approximately assumed being a rigid cylinder, we can use Zimm's equation for the second virial coefficient ${ }^{46,47}$ which is given by

$$
\mathrm{A}_{2}=\pi \mathrm{N}_{\mathrm{a}} \mathrm{d}_{\text {cross }} \frac{\mathrm{I}_{\mathrm{K}}{ }^{2}}{\left(2 \mathrm{M}_{\mathrm{I}_{\mathrm{K}}}\right)^{2}}
$$

which allows us to estimate the cross-sectional diameter $\mathrm{d}_{\text {cross }}$ of the cylinder. Taking the extrapolated values of $A_{2}$ at $M_{I_{K}}=2400,1700$, and 2060 for Cd-tren, Ni-tren, and cuoxam, respectively, one obtains cross-sectional diameter $\mathrm{d}_{\text {cross }}=0.54,0.36$, and $0.52 \mathrm{~nm}$ and for the axial ratios $p=I_{K} / d_{\text {cross }}=27,30$, and 25 , respectively. On average the diameters are $\left\langle\mathrm{d}_{\text {cross }}\right\rangle=0.48 \pm 0.12 \mathrm{~nm}$ and an axial ratio of $\langle p\rangle=27 \pm 7$ with an estimated error of $25 \%$. The data are, of course, rough estimations but well in the range of expectation.

The $\rho=\mathbf{R}_{\mathbf{g}} / \mathbf{R}_{\mathbf{h}}$ Parameter. The last quantity to be discussed is the $\rho=\mathbf{R}_{\mathrm{g}} / \mathbf{R}_{\mathrm{h}}$ parameter which al so gives some indications for the structure of objects in solution. ${ }^{30,48,49}$ The properties result from the fact that the radius of gyration is solely geometrically defined, but the hydrodynamic radius in addition is determined by hydrodynamics interaction among the segments in a coil. Translational diffusion of a macromolecule can often be described by an equivalent sphere that partially can be drained by the solvent. ${ }^{46,50,51} \mathrm{~F}$ or a high segment density, the draining is weak and the hydrodynamic interaction becomes large. For wormlike chains, on the other hand, the solvent can deeply penetrate into the rather open coil, and the sphere radius is small. For impenetrable spheres with a well-defined surface the $\rho$ parameter has a value of $\rho=0.778$. It becomes larger for branched macromolecules $(\rho \approx 1.0$ in the limit of many branching units per macromolecule $\left.{ }^{30}\right)$, increases further for flexible linear chains $(\rho=1.23-1.5)$, and assumes values larger than $\rho=2$ for long cylinders. ${ }^{31}$ Polydispersity and excluded-volume effects increase the value further. 30,31,52 The hydrodynamic radius $R_{h}$ depends only weakly on these quantities, but the radius of gyration is rather sensitive to polydispersity. Within the scatter of experimental accuracy there was no dependence of the $\rho$ parameter on the molar mass. The values in the three solvents are as follows: Cd-tren, $\rho$ $=1.82 \pm 0.14 ;$ Ni-tren, $\rho=2.50 \pm 0.19$; and cuoxam, $\rho$ $=2.51 \pm 0.24$. Accordingly, Cd-tren behaves like a polydisperse Gaussian coil with only weak indications for chain stiffness. From the values for the two other solvents a stronger influence of chain stiffness seemed to be effective. This result is at variance with the actually determined chain stiffness. This leads to the 
conclusion that the coordinatively bound metal complex may have a special contribution to the hydrodynamic interaction that so far is not known to us.

\section{Conclusions}

Cellulose can be dissolved to a molecular level in aqueous metal-based solvents. Most effective is a coordinative binding of the metal complex to the deprotonated olate anions in the $\mathrm{C} 2$ and $\mathrm{C} 3$ position of the anhydro glucose units in the chain. Apparently, coordinative binding occurs on every AGU. The three solvents cuoxam, Ni-tren, and Cd-tren do not differ much in behavior and show good solvent behavior for cellulose. However, the well-known asymptotes for flexible lineare chains were not yet reached. The reason for this may be found in chain stiffness that is about 7-12 times larger than for polystyrene. The number of Kuhn segment lengths per chain exceeded only in a few cases the limit of $\mathrm{N}_{\mathrm{K}}>50$ (indicated by a cross in Figure 12). At this point, according to Fujita, ${ }^{45}$ the influence of excluded vol ume becomes noticeable. This statement is confirmed by the coil interpenetration function $\Psi(\mathrm{z})$ that just at this point started to increase but remained still much smaller than the asymptotic value of 0.269 . Much longer cellulose chains are required before the asymptote can be reached. The chain stiffness is very likely caused by an enhanced strength of the hydrogen bonds between the deprotonated ol ate $\mathrm{O}_{3}{ }^{-}$and the ring oxygen of the next AGU. ${ }^{11}$ In cellulose successive anhydro glucose units are rotated by $180^{\circ}$. Thus, the chain resembles a conformational alternating copolymer with cell obiose as a repeating unit. Thus, the hydrogenbonding system reduces segmental mobility in a similar manner as in ladder polymers.

Acknowledgment. The work was generously supported by the Deutsche Forschungsgemeinschaft under the scheme Cellulose and Cellulose Derivatives.

\section{Appendix: Notation and Abbreviations}

Throughout this paper notations and abbreviations are used which are not common in polymer science. A short explanation is listed here.

Alcohols are commonly denoted by endings of -ol; the corresponding deprotonated al cohol is denoted as ol ate. Example: methanol and methanolate, respectively. If two $\mathrm{OH}$ groups are deprotonated, the compound is called diolate

In a short-hand writing the organic ligands are abbrevated as en $=$ ethylenediamine and - tren $=$ tris(2-aminoethyleneamine) $=\mathrm{N}\left(\mathrm{CH}_{2}-\mathrm{CH}_{2}-\mathrm{NH}_{2}\right)_{3}$. Ethylenediamine is a bidentate and tris(2-aminoethylene amine) a tetradentate ligand (compare Figures 1 and 2).

Complexes are called homoleptic when all ligands in a complex are the same in composition. Compl exes with different kinds of ligands are denoted as heteroleptic.

\section{References and Notes}

(1) (a) Staudinger, H. Diehochmolekularen organischen Verbindungen-Kautschuk und Cellulose; Springer: Berlin 1932. (b) Arbeitserinnerungen; Hütig: Heidel berg, 1961 (English transIation: From Organic Chemistry to Macromolecules; Wiley: New York, 1970).

(2) Staudinger, H. Fritschi, J . Helv. Chim. Acta 1922, 5, 785.
(3) Fengel, D.; Wegener, G. Wood; de Gruyter: Berlin 1989.

(4) (a) Schulz, L.; Burchard, W.; Dönges, R. In Heinze, T. J ., Glasser, W. G., Eds.; Cellulose Derivatives, Modifications Characterization and Nanostructures; ASC Symp. Ser. 1998, 688, 218. (b) Schulz, L.; Seger, B.; Burchard, W. Macromol. Chem. Phys., in press.

(5) Schweizer, E. J . J . Prakt. Chem. 1857, 72, 109.

(6) Fuchs, R.; Habermann, N.; Klüfers, P. Angew. Chem., Int. Ed. Engl. 1993, 32, 852.

(7) Burchard, W.; Habermann, N.; Klüfers, P.; Seger, B.; Wilhelm, U. Angew. Chem., Int. Ed. Engl. 1994, 33, 884.

(8) Burchard, W.; Seger, B. Macromol. Symp. 1994, 83, 291.

(9) Seger, B.; Aberle, T.; Burchard, W. Carbohydr. Polym. 1996, $31,105$.

(10) (a) J ayme, G. Tappi 1962, 44, 299. (b) New Solvents. In Celluloseand Cellulose Derivatives; Bikales, N. M., Segal, L., Eds.; Wiley: New York, 1971; pp 381-410.

(11) (a) Burger, J .; Kettenbach, G.; Klüfers, P. Macromol. Symp. 1995, 99, 113. (b) Kettenbach, G.; Klüfers, P.; Mayer, P. Macromol. Symp. 1997, 120, 291.

(12) (a) Gardner, K. H.; Blackwell, J . Biopolymers 1974, 13, 1975. (b) Kolpak, P. J .; Blackwell, J. Macromolecules 1976, 9, 273.

(13) Normann, W. Chem. Ztg. 1906, 30, 584.

(14) Klaassen, M.; Klüfers, P. Z. Anorg. Allg. Chem. 1993, 619, 661.

(15) Traube, W. Ber. Dtsch. Chem. Ges. 1921, 54, 3320.

(16) Ballauff, M.; Kettenbach, G.; Hanemann, O.; Klüfers, P. Chem. Eur. J ., in press.

(17) Ballauff, M.; Hanemann, O. Macromol ecules 1997, 30, 7638.

(18) Huglin, M. B. Light Scattering from Polymer Solutions; Academic Press: London, 1972; Chapter 6, pp 165-331.

(19) Strazielle, C. Light scattering in mixed solvents. In Huglin, M. B., Ed.; Light Scattering from Polymer Solutions; Academic Press: London, 1972.

(20) Compare also: Kirkwood, J . G.; Goldberg, R. J . J . Chem. Phys. 1950, 18, 54. Stockmayer, W. H. J . Chem. Phys. 1950 $18,58$.

(21) Gralén, N. Ph.D. Thesis, University of Uppsala, 1944.

(22) Burchard, W.; Husemann, E. Makromol. Chem. 1961, 44$46,358$.

(23) Dandliker, W. B.; Kraut, J . J . Am. Chem. Soc. 1956, 78, 2380.

(24) Williams, G.; Watts, O. C. Someaspects of die ectric relaxation of amorpheous polymers. In Diehl, P., Fluck, E., Kosfeld, R., Eds.; NMR: Basis Principles and Progress; Springer: Berlin 1971; Vol. 4, p 271

(25) Berne, B. J .; Pecora, R. Dynamic Light Scattering; Wiley: New York, 1976

(26) Brown, W., Ed. Dynamic Light Scattering: Methods and Some Applications; Clarendon Press: Oxford, 1993.

(27) Brown, W., Ed.; Light Scattering: Principles and Development; Clarendon Press: Oxford 1996.

(28) Klemm, D.; Dugarmaa, S. Manuscript in preparation.

(29) Bantle, S.; Schmidt, M.; Burchard, W. Macromolecules 1982 15, 1604.

(30) Burchard, W.; Schmidt, M.; Stockmayer, W. H. Macromolecules 1980, 13, 1265 .

(31) Schmidt, M.; Stockmayer, W. H. Macromolecules 1984, 17, 509.

(32) Seger, B. Ph.D. Thesis, University of Freiburg, 1996.

(33) Schulz, G. V.; Blaschke, F. J . Prakt. Chem. 1941, 148, 136.

(34) De Gennes, P.-G. Scaling Concepts in Polymer Physics; Cornell University Press: Ithaca, NY, 1979.

(35) Freed, K. F. Renormalization Theory of Macromolecules; Wiley: New York, 1987

(36) Flory, P. J . Statistical Mechanics of Chain Molecules; Wiley: New York, 1969.

(37) Koyama, R. J . Phys. Soc. J pn. 1973, 34, 1029.

(38) Denkinger, P.; Burchard, W. J . Polym. Sci., Polym. Phys 1991, B29, 539.

(39) Dolega, R. Special Development of Laboratory Burchard, University of Freiburg, 1990

(40) Casassa, E. F. J . Chem. Phys. 1955, 23, 596.

(41) Holtzer, A. J . Polym. Sci. 1955, 17, 432.

(42) Schmidt, M.; Paradossi, G.; Burchard, W. Makromol. Chem., Rapid Commun. 1985, 6, 767.

(43) Benoit, H.; Doty, P. J . Phys. Chem. 1953, 57, 958.

(44) Schmidt, M. Macromolecules 1984, 17, 553.

(45) Fujita, H. Macromolecules 1988, 21, 129.

(46) Yamakawa, H. Modern Theory of Polymer Solutions; Harper \& Row: New York, 1971.

(47) Zimm, B. H. J . Chem. Phys. 1948, 16, 1099. 
(48) The reciprocal parameter $\xi=1 / \rho=R_{h} / R_{g}$ was introduced for a linear chain by Tanford (ref 45) and is used occasionally also for the characterization of other architectures.

(49) Tanford, C. Physical Chemistry of Macromolecules; Wiley: New York, 1967; p 344.

(50) Kirkwood, J . G.; Riseman, J . J . Phys. Chem. 1948, 16, 565.
(51) Debye, P.; Bueche, A. M. J . Chem. Phys. 1948, 16, 573.

(52) Burchard, W. Combined Static and Dynamic Light Scattering. In Brown, W., Ed.; Light Scattering: Principles and Some Applications; Clarendon Press: Oxford, 1996.

MA991893M 